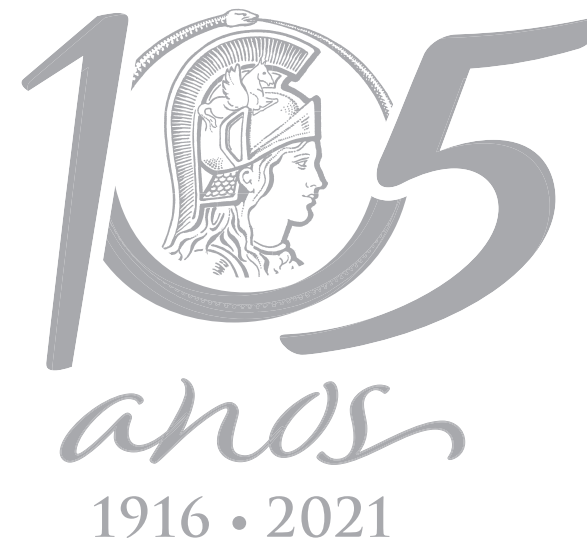

$1916 \cdot 2021$

\title{
GEOSCIENCES
}

\section{Soil pockets phosphatization and chemical weathering of sites affected by flying birds of Maritime Antarctica}

\author{
DAVÍ DO VALE LOPES, FÁBIO S. DE OLIVEIRA, JOSÉ JOÃO L.L. DE SOUZA, MARIANA \\ DE RESENDE MACHADO \& CARLOS ERNESTO G.R. SCHAEFER
}

\begin{abstract}
The majority of ornithogenic soils studied in Antarctica focus on the influence of penguins, wherever little reports evaluated the influence of flying birds on soil genesis. This study aimed to characterize the morphologic, chemic, physic, mineralogic, and micromorphologic ornithogenic soil pockets influenced by flying birds in Snow Island, Maritime Antarctica. Fifteen soil pockets were selected, described, sampled and analyzed, these sites constitute the main areas with intense long-term terrestrial biological activity in Snow Island. In order to investigate the impact of phosphatization, we compared the soil pockets with the surrounding soils and soils affected by penguins. Zone of phosphatization have a high concentration of $\mathrm{P}, \mathrm{K}$, and $\mathrm{Ca}$. The XRD patterns for the clay fraction of ornithogenic soils show that phosphate minerals are the main crystalline phases (leucophosphite, minyulite, fluorapatite, and apatite). We show that even under typical periglacial conditions, sites influenced by flying birds present active chemical weathering processes. The phosphatization release exchangeable bases and accelerate mineralogical and micromorphological transformations in soils. Under the current global warming trend and expected sea-level rise, the ornithogenic environments are susceptible to accelerated erosion rates and a great part of these hotspots may be lost for the open sea.
\end{abstract}

Key words: Global warming, nutrients hotspots, Ornithogenic soil, secondary minerals.

\section{INTRODUCTION}

Ornithogenic soils are composed by mineral and organic materials influenced by birds (Ugolini 1972, Simas et al. 2007). They often have a high content of gravels that has been transported by birds and are identified by other features such as bones, carcasses, eggshells, and feathers (IUSS Working Group WRB 2014). Ornithogenic soils from Maritime Antarctic are different from those of the Continent (Tatur \& Myrcha 1984, Souza et al. 2014). In wetter climates, the substances leached/washed from the decomposition of guano react with the underlying substrate to form a broad phosphatizaed zone (Tatur
\& Myrcha 1984, Simas et al. 2007, Lopes et al. 2021a). The ornithogenic soils of this region are unique in Antarctica and represent important sites where phosphatization is the main soilforming process (Simas et al. 2007, Daher et al. 2019, Lopes et al. 2019). These conditions favor the formation of deep and clayey soils. However the genesis, processes, and transformation systems in phosphatized environments of flying birds are poorly known, compared with those under penguins.

Ornithogenic soils are composed by mineral and organic materials influenced by birds (Ugolini 1972, Simas et al. 2007). They often have a high 
content of gravels that has been transported by birds and are identified by other features such as bones, carcasses, eggshells, and feathers (IUSS Working Group WRB 2014). Ornithogenic soils from Maritime Antarctic are different from those of the Continent (Tatur \& Myrcha 1984, Souza et al. 2014). In wetter climates, the substances leached/washed from the decomposition of guano react with the underlying substrate to form a broad phosphatizaed zone (Tatur \& Myrcha 1984, Simas et al. 2007, Lopes et al. 2021a). The ornithogenic soils of this region are unique in Antarctica and represent important sites where phosphatization is the main soilforming process (Simas et al. 2007, Daher et al. 2019, Lopes et al. 2019). These conditions favor the formation of deep and clayey soils. However the genesis, processes, and transformation systems in phosphatized environments of flying birds are poorly known, compared with those under penguins.

Birds activity during the short austral summer in Maritime Antarctica promotes sealand interactions, transference of nutrients and organic matter (Simas et al. 2007). Large amounts of guano deposited by marine birds accumulate in ice-free areas (Simas et al. 2007, Daher et al. 2019), and these ornithogenic sites constitute the most important carbon reservoirs in Antarctic terrestrial ecosystems (Ugolini 1972, Simas et al. 2007), where vegetation growth is favorable (Tatur \& Myrcha 1989, Cocks et al. 1999, Michel et al. 2006). These unique soils have granular and sub-rounded structures, mobilization of phosphates (illuviation) and secondary mineral formation, characterizing the phosphatization process (Simas et al. 2007).

Recent studies in periglacial environments aimed to understand how chemical weathering processes are associated with ornithogenic areas in Antarctica (Ugolini 1972, Tatur \& Myrcha 1984, 1989, Tatur 1989, Tatur et al. 1997, Cocks et al. 1999, Michel et al. 2006, Simas et al. 2007, Pereira et al. 2013, Schaefer et al. 2017, Daher et al. 2019, Lopes et al. 2021a, Rodrigues et al. 2021). The majority of ornithogenic soils studies focus on the influence of penguins (Ugolini 1972, Tatur \& Myrcha 1984, 1989, Tatur et al. 1997, Simas et al. 2007, Schaefer et al. 2017), wherever little reports evaluated the influence of flying birds on soil genesis (Cocks et al. 1999).

This study purposed to analyze the phosphatization as a soil-forming process and unusual chemical weathering processes of sites affected by flying birds in the President Head Peninsula, Snow Island, Maritime Antarctica. These results enhance the debate on phosphatization and chemical weathering in periglacial environments and provide a baseline for the degree of ornithogenic influence with new approaches. Morphological, physical, chemical, mineralogical, and micromorphological characteristics of soils affected by flying birds were studied, allowing to improve the definitions, qualifiers, and diagnostic criteria of ornithogenic soils by the international WRB and Soil Taxonomy classification systems.

\section{MATERIALS AND METHODS}

\section{Study area}

President Head Peninsula (PHP) is located in the eastern sector of Snow Island (62 $42^{\prime} 54.7$ "S - 61 $14^{\circ}$ '03.7"W), South Shetlands Archipelago, Maritime Antarctica (Figure 1). The Peninsula extends for $2.6 \mathrm{~km}$ in the east-northeast direction. The lithology varies from igneous to sedimentary rocks (basalts, andesites, tuffs, siltstones, mudstones, and sandstones) (Smellie et al. 1984, Torres et al. 1997).

The geomorphology of PHP reveals differences between paraglacial and periglacial landforms, and the area is dominated by a broad central plateau surrounded by talus slope and 

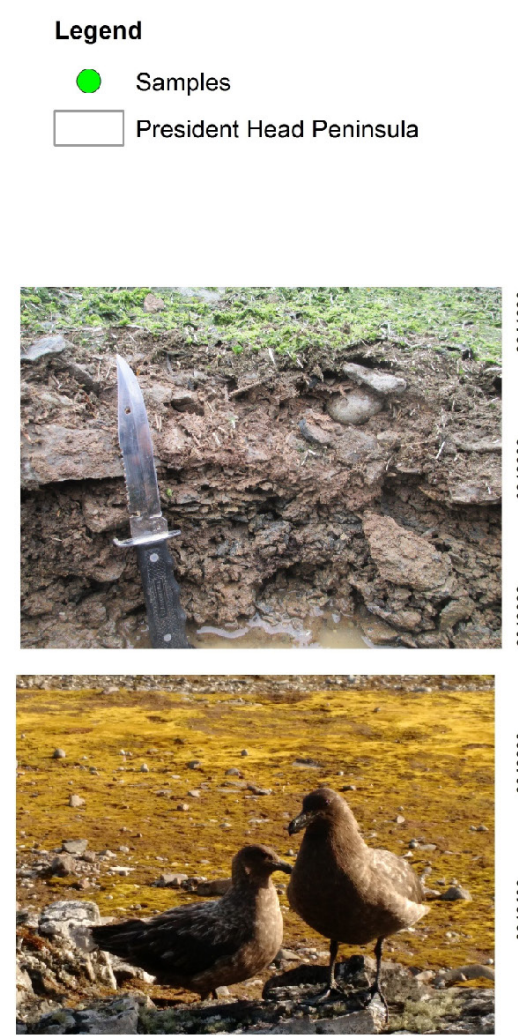
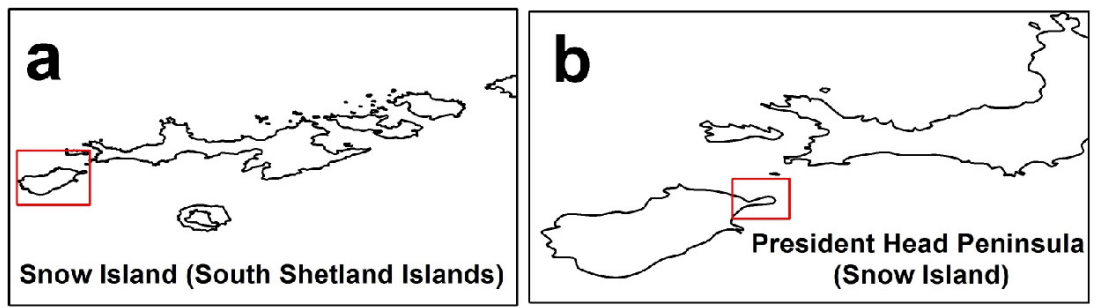

589600

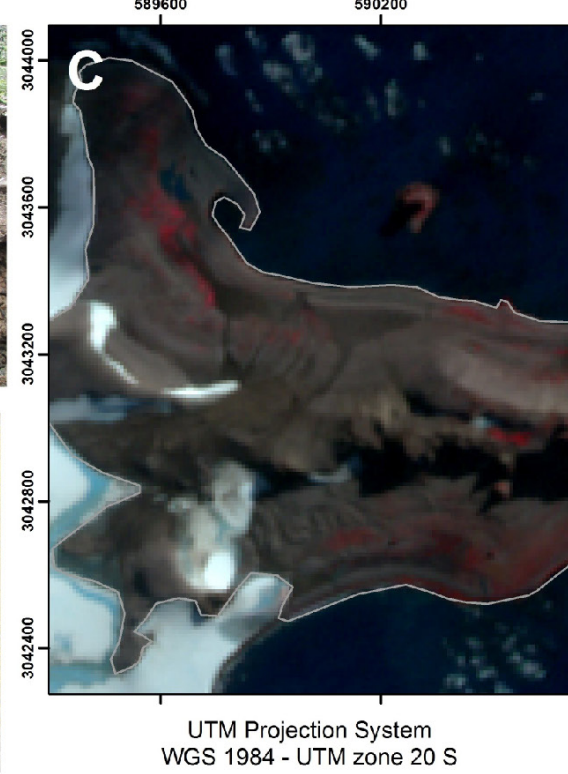

592000

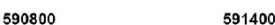

591400

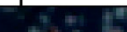

591400

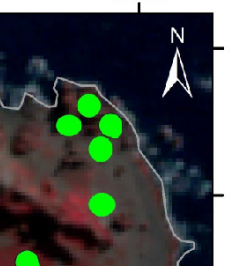

Figure 1. Localization of President Head Peninsula (c), Snow Island (b), South Shetlands Islands (a).

extensive coastal plains, where different levels of marine terraces occur. Lopes et al. (2021 in preparation) stratified President Head Peninsula into two landscape domains: 1) The sedimentary Paraglacial sector, at the western part of the Island, forming a dissected plateau, underlain by a sequence of Cretaceous sediments. These, active meltwater channels strongly erode this domain. 2) The igneous Periglacial sector, at the eastern part of the Island, with a flat-top plateau at about up to $70 \mathrm{~m}$ a.s.l., and permafrost and patterned ground above $50 \mathrm{~m}$ a.s.l..

During the summer 2017 expedition to Snow Island, we observed the local fauna, including numerous seal colonies, especially elephant seals and occasional penguins at the Holocene Beach. Other birds, like skuas and giant petrels have abundant nesting sites on the coastal rocky outcrops, where soil pockets occur in microdepressions.

\section{Sampling sites}

Fifteen (15) surface soil pockets samples on coastal rock outcrops were selected during the 2017 austral summer in PHP. These sites constitute the main areas with intense longterm terrestrial faunal activity in Snow Island. Compound surface samples $(0-20 \mathrm{~cm})$ of present-day petrels and skuas nests were collected. The sampling design aimed to represent the influence of flying birds in the chemical weathering in the coastal domain.

\section{Morphological, physical and chemical analyses}

Samples were air-dried and sieved through a $2 \mathrm{~mm}$ sieve (Embrapa 1997). Sand, silt, and 
clay content were determined by the pipette method after dispersion with $0.1 \mathrm{M} \mathrm{NaOH}$. Soil $\mathrm{pH}$ was determined in distilled water with a glass electrode in a 1:2.5 suspension ( $\mathrm{v} / \mathrm{v}$ soil and water) (Embrapa 1997).

The potential acidity $(\mathrm{H}+\mathrm{Al})$ was extracted by $1 \mathrm{M}$ ammonium acetate solution at $\mathrm{pH}$ 7.0. The content of exchangeable $\mathrm{Ca}^{2+}, \mathrm{Mg}^{2+}$, and $\mathrm{Al}^{3+}$ were determined in a $1 \mathrm{M} \mathrm{KCl}$ extract. Exchangeable $\mathrm{K}^{+}$and $\mathrm{Na}^{+}$were determined after Melhich-1 extraction. Phosphorus content (PM) was determined by a Mehlich-1 extraction solution. All chemical analyses complied with Embrapa (1997). The total organic carbon (OC) was determined by wet combustion (Yeomans \& Bremner 1988).

\section{Mineralogical and micromorphological analyses}

The mineralogy of the clay fraction was determined by X-ray diffraction (XRD) on powdered samples. The XRD employed a PANalytical $X^{\prime}$ Pert Pro Diffractometer using Co $\mathrm{Ka}$ radiation $(40 \mathrm{kV}, 30 \mathrm{~mA})$ in a range of $5-70^{\circ} 2 \theta$ at an increment of $0.008^{\circ} 2 \theta$ steps per second. The clay fraction was submitted to the following treatments before $X$-ray analyzes: solubilize oxides and hydroxides with dithionite-citratebicarbonate $(\mathrm{DCB}), \mathrm{Mg}$ saturation $\left(\mathrm{MgCl}_{2}\right)$, glycerol saturation, $\mathrm{K}$ saturation $(\mathrm{KCl})$ at room temperature, heating of $\mathrm{K}$-saturated samples to $350^{\circ} \mathrm{C}$, and then to $550^{\circ} \mathrm{C}$.

Undisturbed soil blocks were sampled (0$10 \mathrm{~cm}$ ), dried at $50{ }^{\circ} \mathrm{C}$ and impregnated with cryetic resin. Impregnated samples were cut $(0.5$ $\mathrm{cm}$ thickness) using a diamond saw, polished and mounted onto glass slides. Thin sections of soils were described in a Zeiss microscope fitted with a digital camera. The micromorphological description was based on Stoops (2003) and Stoops et al. (2010).

\section{Statistical analyses}

Descriptive statistical analyses (mean, median, maximum, minimum, percentile 10 and 90, coefficient of variation, skewness and kurtosis) were performed. We used 17 samples of ornithogenic soils derived from penguin activities studied by Simas et al. (2007), Pereira et al. (2013), Simas et al. (2015), Daher et al. (2019) and 15 samples (Table I) of ornithogenic soils affected by airborne birdlife nesting of Snow Island, all located in Maritime Antarctica. The whole procedure to examine the descriptive statistical analysis was performed using the software STATISTICA (version 8.0).

In order to investigate the impact of phosphatization in the study area, we compared the soil pockets with the surrounding soils, presenting results of two non-ornithogenic reference pedons, respectively on marine terrace and upper plateau.

\section{RESULTS}

\section{Morphological, physical and chemical properties}

The ornithogenic soil pockets of PHP showed a mean of $46 \%$ of gravels (Table I), with textural range from clay loam to loamy sand, while sandy clay loam is the main soil texture (Table I). In general, soils have dark to olive gray colors (Table I). The soils have high mean contents of sand (54.6\%), followed by $20 \%$ clay and $16.8 \%$ silt. Clay values are 1.65 higher than non-ornithogenic soils (Table I).

Ornithogenic soil pockets have an acidic reaction, with $\mathrm{pH}$ (water) ranging from 4.2 to

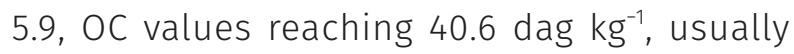
eutrophic (mean 54.6\%), with high levels of Melich-1 extractable-P (4503.7 $\mathrm{mg} \mathrm{dm}^{-3}$ ) and high $\mathrm{Na}^{+}$(mean $739.2 \mathrm{mg} \mathrm{dm}{ }^{-3}$ ) (Table II). The mean contents of exchangeable $\mathrm{Ca}\left(7.5 \mathrm{cmol}_{\mathrm{c}} \mathrm{dm}^{-3}\right), \mathrm{K}$ 


\begin{tabular}{|c|c|c|c|c|c|c|c|c|c|c|c|c|c|c|c|c|c|c|c|c|c|}
\hline 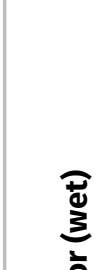 & 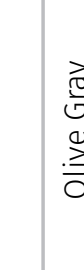 & 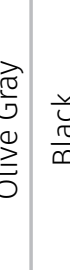 & 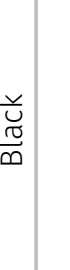 & 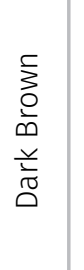 & $\begin{array}{l}\sqrt{0} \\
0 \\
\frac{0}{\sqrt{0}} \\
0 \\
0 \\
\frac{1}{0} \\
>\end{array}$ & $\begin{array}{l}\overrightarrow{0} \\
0 \\
0 \\
\frac{1}{0} \\
0 \\
\frac{\lambda}{0} \\
>\end{array}$ & 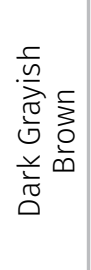 & $\begin{array}{l}\frac{\pi}{0} \\
0 \\
\frac{1}{ \pm} \\
0 \\
0 \\
\frac{\lambda}{0} \\
3\end{array}$ & $\begin{array}{l}\stackrel{\ddot{U}}{\frac{\pi}{D}} \\
\frac{0}{\infty}\end{array}$ & 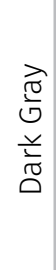 & 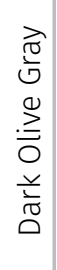 & $\begin{array}{l}\frac{\pi}{0} \\
0 \\
\frac{1}{0} \\
0 \\
0 \\
\frac{\lambda}{0} \\
>\end{array}$ & 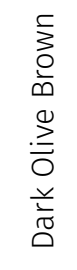 & 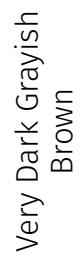 & 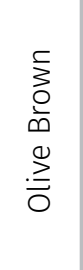 & 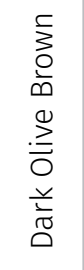 & 1 & & 1 & 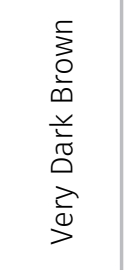 & 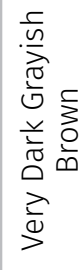 \\
\hline 30 & $\exists$ & $\frac{v}{f} \bar{\gamma}$ & $\grave{v}$ & $\frac{m}{m}$ & $\frac{r}{m}$ & $\frac{\Sigma}{m}$ & $\stackrel{\sim}{\triangleleft}$ & $\frac{\Sigma}{m}$ & $\stackrel{\leftarrow}{\stackrel{5}{\sim}}$ & $\frac{5}{f}$ & $\frac{N}{m}$ & $\frac{\Sigma}{m}$ & $\frac{m}{m}$ & $\frac{N}{m}$ & $\frac{m}{f}$ & $\frac{m}{m}$ & ' & & 1 & $\stackrel{\curvearrowright}{\curvearrowright}$ & $\frac{N}{m}$ \\
\hline & むু & 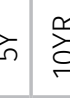 & or & 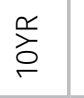 & 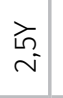 & 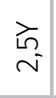 & 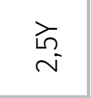 & え & え & え & え & $\stackrel{\grave{L}}{i}$ & 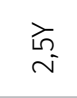 & 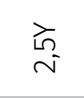 & 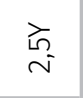 & 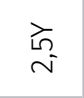 & 1 & & ' & $\stackrel{\alpha}{\partial}$ & 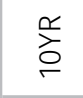 \\
\hline$\frac{\frac{\lambda}{0}}{\frac{2}{0}}$ & 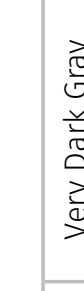 & 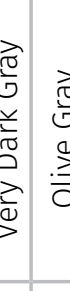 & 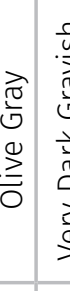 & 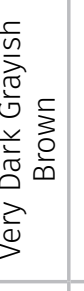 & 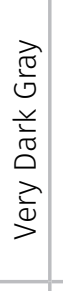 & $\begin{array}{l}\vec{a} \\
\frac{0}{0} \\
\frac{v}{i} \\
0 \\
\frac{1}{0} \\
\frac{2}{3}\end{array}$ & 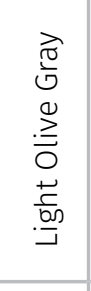 & 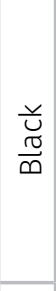 & 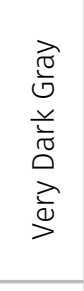 & 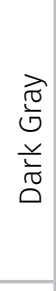 & 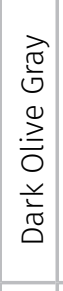 & 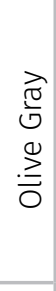 & 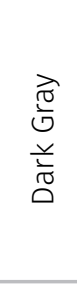 & $\begin{array}{l}\overrightarrow{0} \\
\stackrel{0}{0} \\
0 \\
\stackrel{\Xi}{0}\end{array}$ & 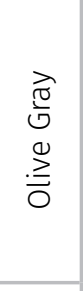 & 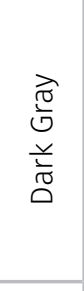 & 1 & & I & 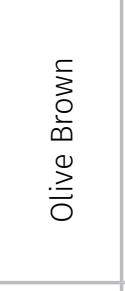 & $\begin{array}{l}\frac{5}{3} \\
\frac{0}{0} \\
\frac{1}{n} \\
\frac{n}{2} \\
\frac{\pi}{0}\end{array}$ \\
\hline$\overline{0}$ & & $\frac{\Sigma}{m} \stackrel{5}{\llcorner}$ & $\frac{N}{n}$ & $\frac{N}{m}$ & $\bar{m}$ & $\frac{\Sigma}{m}$ & $\frac{N}{6}$ & $\underset{\substack{\Sigma \\
\sim}}{\sim}$ & $\frac{\Sigma}{m}$ & $\frac{5}{\lessgtr}$ & $\frac{N}{m}$ & $\stackrel{\sim}{f}$ & $\underset{\Im}{\lessgtr}$ & $\frac{N}{n}$ & $\stackrel{\sim}{\checkmark}$ & $\frac{5}{f}$ & 1 & & ' & $\frac{m}{f}$ & $\frac{n}{n}$ \\
\hline & むু & ঠ む & $\succsim$ & 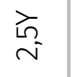 & 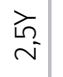 & $\succsim$ & $\succsim$ & $\succsim$ & $\succsim$ & え & え & え & え & $\succsim$ & $\succsim$ & え & 1 & & 1 & $\stackrel{\succsim}{\longleftarrow}$ & $\stackrel{\succsim}{\sim}$ \\
\hline 冚 & & 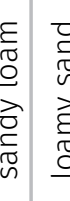 & 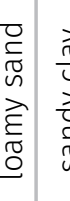 & 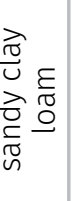 & $\begin{array}{l}\frac{\varepsilon}{0} \\
0 \\
0 \\
\overrightarrow{0} \\
\frac{0}{0} \\
\omega\end{array}$ & $\begin{array}{l}\frac{E}{0} \\
0 \\
0 \\
\overrightarrow{0} \\
0 \\
\overline{0} \\
0\end{array}$ & 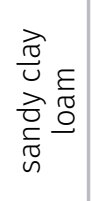 & 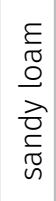 & 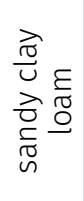 & $\begin{array}{l}\frac{E}{0} \\
\frac{0}{O} \\
\frac{\pi}{U}\end{array}$ & 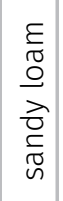 & 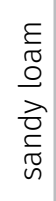 & 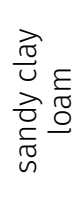 & 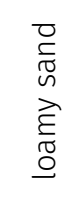 & 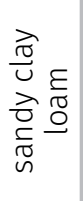 & 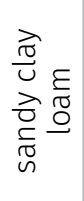 & ' & & ' & 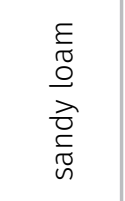 & 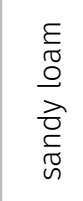 \\
\hline 츤 & & 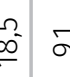 & $\bar{\sigma}$ & $\stackrel{\tilde{\sim}}{\tilde{\sim}}$ & $\begin{array}{l}\stackrel{0}{\digamma} \\
\stackrel{1}{*}\end{array}$ & $\begin{array}{l}\infty \\
\stackrel{\infty}{\sim}\end{array}$ & $\stackrel{\bullet}{\sim}$ & $\stackrel{m}{\sigma}$ & 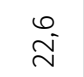 & $\stackrel{\AA}{i}$ & $\bar{\sigma}$ & $\bar{\sigma}$ & $\begin{array}{l}\stackrel{0}{\sim} \\
\stackrel{n}{\sim}\end{array}$ & $\check{F}$ & $\stackrel{\circ}{\stackrel{N}{N}}$ & ¿ & $\begin{array}{l}0 \\
\text { ¿ }\end{array}$ & $\hat{\kappa}$ & $\stackrel{m}{\sigma}^{2}$ & $\overline{\check{T}}$ & $\bar{\cong}$ \\
\hline$\stackrel{ \pm}{n}$ & 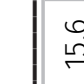 & $\stackrel{e}{e}$ & $\hat{\imath}$ & $\begin{array}{l}a \\
\tilde{b}^{-}\end{array}$ & a. & $\stackrel{\sim}{F}$ & 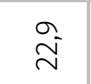 & $\hat{夭}$ & $\stackrel{\overbrace{}}{\tilde{N}}$ & $\bar{s}$ & $\overline{\mathrm{s}}$ & $\stackrel{\curvearrowright}{\tilde{N}}$ & $\stackrel{m}{F}$ & $\stackrel{\infty}{m^{-}}$ & $\stackrel{\llcorner\Omega}{\llcorner}$ & $\bar{\sigma}$ & $\begin{array}{l}\infty \\
\sigma^{-}\end{array}$ & $a^{m}$ & $\begin{array}{l}\dot{0} \\
\stackrel{\sim}{\sim}\end{array}$ & ã & $\bar{\infty}^{-}$ \\
\hline 害勇 & ๙ิ & \begin{tabular}{c}
\multicolumn{2}{c}{} \\
$\sigma$ \\
\end{tabular} & $\stackrel{\Omega}{\digamma}$ & $\stackrel{m}{\stackrel{\sim}{\sim}}$ & $\hat{\sigma}^{\circ}$ & $\hat{\sigma}$ & $\stackrel{\sim}{\infty}$ & aे & $\Sigma$ & $\begin{array}{l}\stackrel{\sim}{\circ} \\
\stackrel{\circ}{\circ}\end{array}$ & $\begin{array}{l}\mathcal{L}^{-} \\
\infty^{-}\end{array}$ & $\begin{array}{l}\infty \\
0^{-}\end{array}$ & $\begin{array}{l}\stackrel{\llcorner}{\infty} \\
\infty^{-}\end{array}$ & $\stackrel{0}{\omega^{\prime}}$ & $\stackrel{+}{\infty}$ & ผี & $\begin{array}{l}0 \\
\infty^{-}\end{array}$ & $\bar{v}$ & $\begin{array}{l}\stackrel{\llcorner}{\Omega} \\
\infty^{-}\end{array}$ & $\begin{array}{l}\omega^{-} \\
\infty^{-}\end{array}$ & $\stackrel{\sim}{\sim}$ \\
\hline 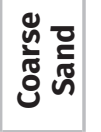 & & $\hat{n}^{-\infty}$ & $\Sigma$ & $\overline{\tilde{m}}$ & $\hat{b}^{\circ}$ & $\begin{array}{l}m \\
\theta^{-}\end{array}$ & $\begin{array}{c}m \\
\text { vo }\end{array}$ & $\stackrel{6}{\tilde{n}}$ & $\stackrel{+}{*}$ & $\stackrel{ \pm}{\sim}$ & 走 & $\underset{f}{\stackrel{\sim}{f}}$ & $\begin{array}{l}0 \\
\dot{H}^{-}\end{array}$ & $\begin{array}{l}\stackrel{2}{2} \\
\stackrel{2}{n}\end{array}$ & $\begin{array}{l}t \\
0 \\
\sigma^{\prime}\end{array}$ & $\overbrace{0}^{1}$ & $\begin{array}{l}0 \\
\text { जే }\end{array}$ & \begin{tabular}{l}
0 \\
\multirow{J}{\pm}{}
\end{tabular} & $\begin{array}{l}0 \\
\text { जే }\end{array}$ & : & $\begin{array}{l}\stackrel{\llcorner}{0} \\
\tilde{L}^{\circ}\end{array}$ \\
\hline $\begin{array}{l}\text { बाँ } \\
\text { जँ }\end{array}$ & & 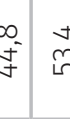 & נָ- & $\begin{array}{l}0 \\
\stackrel{N}{N}\end{array}$ & $\stackrel{m}{\sim}$ & $\stackrel{0}{\infty^{-}} \stackrel{\text { D. }}{\sim}$ & $\stackrel{0}{\stackrel{2}{\Sigma}}$ & $\begin{array}{l}0 \\
\infty \\
\infty^{-}\end{array}$ & $\begin{array}{l}+ \\
\theta^{-}\end{array}$ & $\stackrel{m}{\mathrm{n}^{-}}$ & 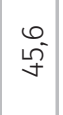 & $\begin{array}{l}0 \\
\frac{-}{4}\end{array}$ & $\begin{array}{l}\text { 足 } \\
\text { 占 }\end{array}$ & $\bar{\sigma}$ & हैं & $\begin{array}{l}\text { बे } \\
\text { ó }\end{array}$ & $\begin{array}{l}0 \\
0^{-} \\
+\end{array}$ & $\check{F}$ & $\begin{array}{l}\sigma \\
\text { aे }\end{array}$ & $\begin{array}{l}m \\
\text { ó } \\
+\end{array}$ & $\begin{array}{l}\stackrel{\sim}{ \pm} \\
\stackrel{ \pm}{\sim}\end{array}$ \\
\hline $\begin{array}{l}\frac{0}{0} \\
\text { हn } \\
\text { ڤn }\end{array}$ & & $\begin{array}{l}\hat{v} \\
\hat{v}\end{array}$ & $\begin{array}{l}n \\
\text { जे }\end{array}$ & $\begin{array}{l}m \\
\text { nे }\end{array}$ & $\begin{array}{l}\dot{ } \\
\text { जे }\end{array}$ & $\begin{array}{l}n \\
\text { ஸे }\end{array}$ & $\begin{array}{l}0 \\
\text { जे }\end{array}$ & $\hat{n}$ & $\begin{array}{l}\infty \\
\text { जे }\end{array}$ & $\begin{array}{l}\hat{a} \\
\hat{n}\end{array}$ & $\begin{array}{l}\text { ㅇ } \\
\text { iे }\end{array}$ & $\begin{array}{l}F \\
\text { in }\end{array}$ & $\begin{array}{l}\stackrel{N}{n} \\
\text { जे }\end{array}$ & $\begin{array}{l}\frac{m}{n} \\
\text { nे }\end{array}$ & $\frac{ \pm}{\text { nे }}$ & $\begin{array}{l}\stackrel{n}{2} \\
\text { nे }\end{array}$ & 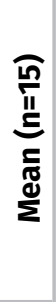 & जे & $\begin{array}{l}\frac{5}{\frac{\pi}{3}} \\
\frac{0}{2}\end{array}$ & 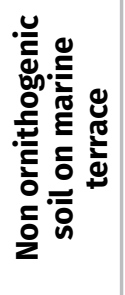 & 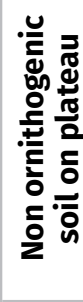 \\
\hline
\end{tabular}


(278.9 $\left.\mathrm{mg} \mathrm{dm}^{-3}\right)$ and $\mathrm{H}+\mathrm{Al}\left(11.8 \mathrm{cmol}_{\mathrm{c}} \mathrm{dm}^{-3}\right)$ were higher in soil pockets than non-ornithogenic soils on the marine terrace (2.0,2.2 and 1.2 times higher, respectively) (Table II).

\section{Comparison between sites affected by flying birds versus penguins}

In Maritime Antarctica, nutrient availability is greatly enhanced by the vast guano deposition promoted by marine birds, and the phosphatization (Michel et al. 2006, Simas et al. 2007, Pereira et al. 2013, Daher et al. 2019, Rodrigues et al. 2019). Descriptive statistics demonstrate that soil pockets affected by flying birds activity show higher mean and median values of clay, P, K, Ca, Mg, BS, CEC and OC, compared to classical ornithogenic soils affected by penguin activity (Table III).

The comparative data demonstrated higher values of $\mathrm{Al}, \mathrm{H}+\mathrm{Al}$, and $\mathrm{Al}$ saturation in penguin nesting areas. High values of $\mathrm{H}+\mathrm{Al}$ can indicate a high organic matters and leaching degree, as reported by Daher et al. (2019). The deeper ornithogenic soils associated with penguins have high losses through leaching, with intense downward water percolation through the soil.

Soil pockets affected by flying birds show the highest extreme values (percentile 10), ornithogenic soils in penguin areas show the highest percentile 90 and coefficient of variation (CV). The shape of a probability distribution was platykurtic for all flying birds variables (except for Al saturation and (EC), and penguins areas (except for $\mathrm{K}$ and OC) (Table III). Skewness (a measure of the asymmetry of the probability distribution) was positive (the distribution is right-skewed) for penguin areas (except for sand) (Table III).

\section{Mineralogical and micromorphological properties}

$X$-ray diffraction patterns of the sand fraction show the presence of mordenite $(0.34,0.40,0.65$ $\mathrm{nm})$, olivine $(0.15,0.18,0.25,0.28,0.40 \mathrm{~nm})$, biotite $(0.21,0.24,0.32 \mathrm{~nm})$, plagioclase $(0.25,0.28,0.32$, $0.40 \mathrm{~nm})$, augite $(0.14,0.17,0.21,0.25,0.28,0.32$ $\mathrm{nm})$, ilmenite $(0.14,0.17,0.19,0.22,0.25,0.27 \mathrm{~nm})$ and traces of apatite (0.27, $0.34 \mathrm{~nm}$ ) (Figure 2).

XRD patterns for the clay fraction show phosphate minerals as the main crystalline phases. The mineralogy was basically composed by leucophosphite $\left(\mathrm{KFe}_{2}\left(\mathrm{PO}_{4}\right)_{2}(\mathrm{OH}) .2\left(\mathrm{H}_{2} \mathrm{O}\right)\right)$ $(0.61,0.55,0.42,0.28 \mathrm{~nm})$, minyulite $\left(\mathrm{KAl}_{2}\left(\mathrm{PO}_{4}\right)_{2}(\mathrm{OH}, \mathrm{F}) .4\left(\mathrm{H}_{2} \mathrm{O}\right)\right)(0.55,0.39,0.34,0.26$ $\mathrm{nm})$, fluorapatite $\left(\mathrm{Ca}_{5}\left(\mathrm{PO}_{4}\right) \mathrm{F}\right)(0.19,0.26 \mathrm{~nm})$ and apatite $\left(\mathrm{Ca}_{10}\left(\mathrm{PO}_{4}\right)_{6}\left(\mathrm{OH}_{2}\right)\right)(0.28,0.26 \mathrm{~nm})$ (Figures 3 and 4). The latter was the only mineral that occurred in all fractions, representing the primary bone-apatite from guano.

In addition, vermiculite (1.51, 0.86, 0.37 $\mathrm{nm})$, plagioclase $(0.24,0.19$ and $0.17 \mathrm{~nm})$, augite $(0.21,0.24,0.29 \mathrm{~nm})$ and biotite (Figures 3 and 4) were also identified in the clay fractions, consistent with previous studies in Maritime Antarctica (Simas et al. 2007, Pereira et al. 2013, Rodrigues et al. 2019). Mica (biotite) has a nonexpendable $\mathrm{d}(001)$ of $1.04 \mathrm{~nm}$, which is not affected by ethylene glycol solvation or heating (Figure 3). Strong 0.22 and 0,24 nm peaks (Figures 3 and 4) that remained stable after heating and glycerol solvation confirm biotite identification. Interestingly, these ornithogenic samples showed a presence of crystalline Feoxyhydroxides, remarkably, goethite $(0.14,0.15$, $0.17,0.22,0.42 \mathrm{~nm}$ ) (Figures 3 and 4), which is unusual for Antarctic soils (Simas et al. 2006). The DCB method was efficient for the removal of iron hydroxides. 


\begin{tabular}{|c|c|c|c|c|c|c|c|c|c|c|c|c|c|c|c|c|c|c|c|c|c|}
\hline 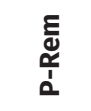 & מִ & 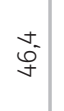 & $\stackrel{0}{i}$ & $\stackrel{\infty}{n} \stackrel{\infty}{n}^{-1}$ & $\widehat{N}$ & $\begin{array}{c}\overline{\sigma o} \\
\sigma^{-}\end{array}$ & $\begin{array}{l}\widehat{0^{-}} \\
{ }^{-}\end{array}$ & $\stackrel{ \pm}{5}$ & $\frac{7}{7}$ & $\stackrel{+}{\stackrel{\infty}{~}}$ & 式 & $\hat{o}^{-0}$ & 穴 & $\hat{o}$ & 文 & 馬 & 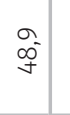 & $\stackrel{2}{a}$ & ô & $\stackrel{\simeq}{\simeq}$ & $\hat{\bar{m}}$ \\
\hline$\breve{~}$ & 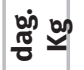 & & 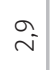 & $\bar{\infty}^{-}$ & $a^{\circ}$ & $\hat{\imath}$ & $\stackrel{ }{F}$ & $\stackrel{m}{i}$ & $\begin{array}{l}0 \\
\tilde{i} \\
\tilde{m}\end{array}$ & $\begin{array}{l}m \\
\infty^{-}\end{array}$ & $\overline{\check{I}}$ & oे & $\begin{array}{l}0 \\
0 \\
\vdots \\
q\end{array}$ & $\stackrel{\sigma}{m}$ & $\bar{\infty}^{-}$ & $\frac{\sigma}{\bar{m}}$ & $\begin{array}{l}\stackrel{\sigma}{f} \\
\dot{f}\end{array}$ & $\begin{array}{l}\stackrel{\sigma}{F} \\
F\end{array}$ & 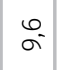 & $\stackrel{\infty}{\wedge}$ & $\stackrel{\bullet}{i}$ \\
\hline$\sum_{\underline{n}}^{00}$ & \multirow{3}{*}{ ¿ீ } & 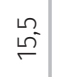 & $\begin{array}{l}\stackrel{0}{m} \\
\mathrm{p}^{2}\end{array}$ & :- & $\stackrel{\leftrightarrow}{\sigma}$ & $\stackrel{\stackrel{L}{\sigma}}{\sigma}$ & $\begin{array}{l}0 \\
\infty^{\circ}\end{array}$ & \begin{tabular}{l}
0 \\
\multirow{1}{\pm}{}
\end{tabular} & 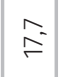 & $\sigma^{\circ}$ & $\hat{\imath}$ & $\bar{\rho}^{-}$ & $\begin{array}{l}0 \\
\infty^{\circ}\end{array}$ & $\stackrel{\infty}{=}$ & है & $\widehat{\infty^{-}}$ & $\stackrel{m}{\sim}$ & $\stackrel{\sim}{f}$ & $\stackrel{\infty}{=}$ & $\stackrel{\stackrel{\llcorner}{\sim}}{\sim}$ & $\stackrel{n}{=}$ \\
\hline 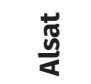 & & $\cong$ & $0_{0}^{\circ}$ & $\stackrel{m}{=}$ & $\stackrel{\infty}{\sim}$ & $\stackrel{\stackrel{n}{\sim}}{\sim}$ & $\stackrel{\llcorner}{\sim}$ & $\stackrel{m}{\sim}$ & 0 & $0^{\circ}$ & :- & O- & \begin{tabular}{l}
0 \\
\multirow{f}{*}{}
\end{tabular} & $\stackrel{n}{\sigma}$ & $\stackrel{\sim}{F}$ & $\stackrel{\sim}{\sim}$ & $\stackrel{+}{m}$ & $\tilde{\omega}^{2}$ & $\stackrel{m}{\sim}$ & $\tilde{\tilde{m}}^{\sim}$ & in \\
\hline ณิ & & 常 & 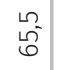 & $\overline{\mathrm{n}}$ & $\stackrel{\llcorner}{\llcorner}$ & 志 & $\overline{\mathrm{n}^{\prime}}$ & 勇 & 苟 & $\begin{array}{l}0 \\
\bar{\theta}^{-}\end{array}$ & $\stackrel{m}{i}$ & $\stackrel{m}{i}$ & 官 & $\begin{array}{l}0 \\
\tilde{m} \\
m\end{array}$ & $\begin{array}{c}0 \\
\dot{m}\end{array}$ & $\begin{array}{c}\infty \\
\infty \\
\infty \\
m\end{array}$ & \begin{tabular}{l}
0 \\
\multirow{1}{*}{}
\end{tabular} & \begin{tabular}{l}
0 \\
\multirow{\leftarrow}{*}{}
\end{tabular} & 走 & $\stackrel{\text { ñ }}{\tilde{n}}$ & $\begin{array}{l}\text { Q } \\
\text { సे }\end{array}$ \\
\hline 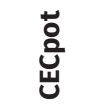 & \multirow{5}{*}{ 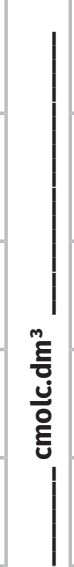 } & సे & $\overline{\mathrm{s}}$ & \begin{tabular}{l}
0 \\
0 \\
\multirow{j}{*}{}
\end{tabular} & $\stackrel{\sim}{\approx}$ & $\stackrel{m}{\sim}$ & 点 & $\hat{\tilde{i}}$ & 灾 & $\begin{array}{l}0 \\
\text { m }\end{array}$ & $\stackrel{m}{\curvearrowright}$ & $\begin{array}{l}\infty \\
\infty^{-}\end{array}$ & 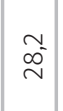 & $\begin{array}{l}\infty \\
\stackrel{\infty}{p}^{-}\end{array}$ & $\stackrel{\infty}{i}$ & $\begin{array}{l}\infty \\
\sigma^{-}\end{array}$ & 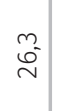 & $\hat{\sigma}$ & $\hat{\mathrm{e}}$ & ث̀ & 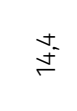 \\
\hline 窇 & & $\stackrel{+}{\stackrel{5}{c}}$ & 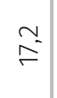 & $\begin{array}{l}0 \\
\text { i }\end{array}$ & 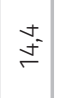 & $\begin{array}{l}0 \\
p^{-}\end{array}$ & $\bar{m}^{-}$ & s. & $\stackrel{+}{\stackrel{5}{=}}$ & $\stackrel{\llcorner}{\sim}$ & $\bar{i}$ & $\begin{array}{l}\stackrel{e}{\rho} \\
\stackrel{\rho}{m}\end{array}$ & $\begin{array}{l}0 \\
\stackrel{0}{\circ}\end{array}$ & $\begin{array}{c}0 \\
\dot{\sigma}\end{array}$ & $\hat{a}$ & $\stackrel{2}{-}$ & $\begin{array}{l}\infty \\
\underset{f}{+}\end{array}$ & ஸ̃ & \begin{tabular}{l} 
\pm \\
\multirow{\leftarrow}{*}{}
\end{tabular} & $\stackrel{m}{\sim}$ & $\stackrel{m}{=}$ \\
\hline$\tilde{\omega}$ & & 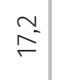 & 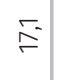 & $\stackrel{\tilde{\vartheta}^{-}}{-}$ & $\begin{array}{l}0 \\
\text { İ }\end{array}$ & $\stackrel{t}{m}$ & 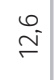 & 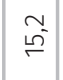 & 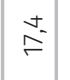 & $\stackrel{n}{i}$ & $\bar{\sim}$ & $\stackrel{\bullet}{\stackrel{\rho}{r}}$ & $\bar{\sigma}^{\circ}$ & $\stackrel{\llcorner}{f}$ & $\begin{array}{l}0 \\
\infty\end{array}$ & $\hat{\imath}$ & $\begin{array}{l}\stackrel{\llcorner}{\sim} \\
\underset{f}{f}\end{array}$ & ثे & \begin{tabular}{l}
0 \\
\multirow{+}{+}{}
\end{tabular} & $\hat{\circ}$ & $\stackrel{\infty}{\circ} \stackrel{-}{\circ}$ \\
\hline $\begin{array}{l}\bar{\varangle} \\
\pm \\
\pm\end{array}$ & & $\underset{\simeq}{\cong}$ & à & ò & $\stackrel{\stackrel{n}{n}}{\Gamma}$ & बे & $\stackrel{\llcorner}{\simeq}$ & $\begin{array}{l}\omega_{0}^{n} \\
\infty^{\prime}\end{array}$ & $a_{\sigma}$ & 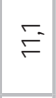 & $\widetilde{\sigma}$ & กี & $\stackrel{\infty^{-}}{\varsigma^{-}}$ & $\sigma^{m}$ & $\stackrel{\sim}{\sigma}$ & $\bar{\tau}$ & $\begin{array}{l}\stackrel{\infty}{\digamma} \\
F\end{array}$ & \begin{tabular}{l}
0 \\
\multirow{f}{*}{}
\end{tabular} & $F$ & $\hat{a}$ & $\stackrel{\stackrel{m}{m}}{*}$ \\
\hline ष & & $\tilde{o}$ & 5 & m. & $0_{0}^{+}$ & ô. & $\begin{array}{l}0 \\
0\end{array}$ & $\tilde{o}$ & O & O- & O- & O & $\stackrel{n}{0}$ & $F$ & $F$ & $\tilde{c}$ & $\stackrel{m}{o}$ & $\stackrel{+}{0}$ & $\tilde{0}$ & $\stackrel{\circ}{\circ}$ & $\stackrel{0}{0}$ \\
\hline$\sum^{\infty}$ & & $\stackrel{+}{m}$ & $\bar{m}$ & $\begin{array}{l}q \\
\dot{f}\end{array}$ & $\bar{m}$ & $\stackrel{0}{i}$ & $\stackrel{i}{i}$ & $\begin{array}{l}0 \\
\text { f }\end{array}$ & $F_{f}$ & $\hat{\sigma}$ & $\stackrel{+}{\circ}$ & $\stackrel{\sim}{\sim}$ & $\stackrel{\curvearrowright}{\sim}$ & $\begin{array}{l}\infty \\
0 \\
0\end{array}$ & $\stackrel{m}{\sim}$ & $\stackrel{m}{\sim}$ & $\bar{m}$ & $\approx$ & $\bar{m}$ & $\stackrel{\circ}{m}$ & $\stackrel{\circ}{\tilde{m}^{\prime}}$ \\
\hline తూ & 1 & $\infty$ & aे & $\stackrel{\infty}{\stackrel{\infty}{\rho}^{-}}$ & 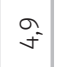 & $\hat{~}$ & $\stackrel{\infty}{\wedge}$ & $\stackrel{m}{\sim}$ & $\approx$ & $\stackrel{+}{F}$ & $\stackrel{\stackrel{n}{F}}{F}$ & $\begin{array}{l}\sigma^{\prime} \\
\infty\end{array}$ & 5 & $\stackrel{0}{-}$ & $\begin{array}{l}0 \\
\text { f }\end{array}$ & $\stackrel{a}{m}$ & $\stackrel{\llcorner}{\wedge}$ & $\stackrel{m}{m}^{-}$ & $\hat{\imath}$ & $\hat{m}$ & हn \\
\hline$\frac{\pi}{2}$ & \multirow{3}{*}{ E⿱ } & \begin{tabular}{l}
0 \\
\multirow{0}{0}{} \\
0 \\
0
\end{tabular} & $\begin{array}{l}\sigma \\
\text { }\end{array}$ & $\begin{array}{l}\hat{\widetilde{J}} \\
\stackrel{0}{\circ}\end{array}$ & 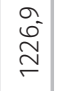 & 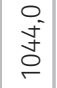 & 志 & $\begin{array}{l}0 \\
0 \\
\\
\end{array}$ & 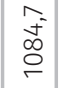 & $\begin{array}{l}\stackrel{\alpha}{\infty} \\
\stackrel{\infty}{\kappa}\end{array}$ & $\begin{array}{l}\sim \\
\stackrel{0}{0} \\
\infty\end{array}$ & 孛 & 告- & \begin{tabular}{|l|}
$\stackrel{n}{\tilde{m}}$ \\
$\stackrel{m}{m}$
\end{tabular} & 㐫 & $\begin{array}{l}\infty \\
\tilde{m} \\
\tilde{m}\end{array}$ & ڤ̃ & ڤ̀- & $\begin{array}{l}\text { o. } \\
\text { 员 }\end{array}$ & $\begin{array}{l}\text { Oे } \\
\text { ○े } \\
\infty\end{array}$ & $\begin{array}{l}0 \\
\stackrel{0}{0} \\
m\end{array}$ \\
\hline$\underline{\simeq}$ & & $\begin{array}{l}\text { :- } \\
\text { oे } \\
\text {. }\end{array}$ & $\begin{array}{l}0 \\
0 \\
0 \\
0 \\
0\end{array}$ & ০े & $\begin{array}{l}0 \\
\stackrel{0}{\circ} \\
\stackrel{\infty}{\sim}\end{array}$ & Oे & $\begin{array}{l}\stackrel{0}{a} \\
\stackrel{a}{\sim}\end{array}$ & $\begin{array}{l}0 \\
\stackrel{\text { ปे }}{ }\end{array}$ & $\begin{array}{l}0 \\
0 \\
0 \\
0 \\
m\end{array}$ & 엄 & $\begin{array}{l}\circ \\
\stackrel{\circ}{0} \\
\stackrel{-}{2}\end{array}$ & 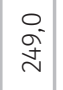 & Oे & $\begin{array}{l}\stackrel{0}{\infty} \\
\stackrel{\infty}{\rightleftharpoons}\end{array}$ & $\begin{array}{l}0 \\
\infty \\
\infty \\
\infty \\
m\end{array}$ & $\begin{array}{l}\text { O- } \\
\text { oे } \\
\text { m }\end{array}$ & $\begin{array}{l}\stackrel{\alpha}{\alpha} \\
\stackrel{\infty}{\sim}\end{array}$ & $\underset{\infty}{\mp}$ & 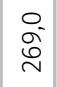 & 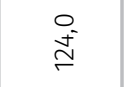 & 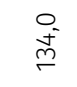 \\
\hline a & & 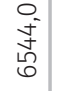 & 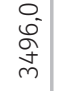 & $\begin{array}{l}0 \\
\stackrel{i}{i} \\
\stackrel{N}{R}\end{array}$ & 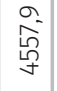 & 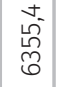 & 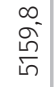 & 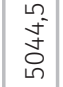 & 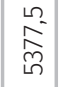 & $\begin{array}{c}\tilde{N} \\
\tilde{N} \\
\stackrel{0}{n}\end{array}$ & 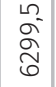 & 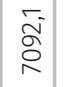 & $\begin{array}{l}a \\
\tilde{j} \\
\tilde{\delta} \\
\tilde{m}\end{array}$ & 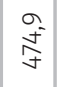 & $\begin{array}{l}m \\
\tilde{O} \\
\tilde{n} \\
\end{array}$ & $\begin{array}{l}\underset{\mathcal{f}}{\tilde{S}} \\
\infty\end{array}$ & $\begin{array}{l}\hat{\text { }} \\
\text { 命 }\end{array}$ & $\begin{array}{l}\bar{D} \\
\stackrel{\sim}{\sim}\end{array}$ & $\begin{array}{l}\infty \\
0 \\
0 \\
\\
\end{array}$ & $\begin{array}{l}\infty \\
\stackrel{\infty}{\infty^{-}}\end{array}$ & 官 \\
\hline 동 $\overline{\underline{y}}$ & & \begin{tabular}{l}
0 \\
\multirow{5}{*}{}
\end{tabular} & $\stackrel{\infty}{m^{\prime}}$ & $\underset{f}{+}$ & $\begin{array}{l}0 \\
\text { f }\end{array}$ & \begin{tabular}{l}
0 \\
\multirow{5}{*}{}
\end{tabular} & $\hat{n^{\circ}}$ & 寺 & $\begin{array}{l}\infty \\
+ \\
f^{-}\end{array}$ & m. & ธี & $\stackrel{M}{n}^{2}$ & $\stackrel{\infty}{m}$ & $\stackrel{+}{m}$ & $\bar{m}$ & $\stackrel{\stackrel{\sigma}{m}}{ }$ & 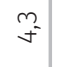 & $\hat{o}$ & $\stackrel{+}{+}$ & F & $\bar{f}$ \\
\hline I & & 今' & \begin{tabular}{l}
$\infty$ \\
\multirow{f}{*}{}
\end{tabular} & \begin{tabular}{l}
$\infty$ \\
\multirow{f}{*}{}
\end{tabular} & $\stackrel{m}{f}$ & เో & $\stackrel{+}{+}$ & $\stackrel{m}{n}^{2}$ & is & ஸे & $\stackrel{\infty}{\llcorner}$ & $\omega^{\infty}$ & f & $\stackrel{+}{+}$ & $\stackrel{\sim}{f}$ & $\begin{array}{l}\infty \\
f^{-}\end{array}$ & í & : & $\begin{array}{l}\infty \\
f^{-}\end{array}$ & $\bar{f}^{-}$ & : \\
\hline 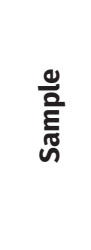 & & $\bar{i}$ & $\begin{array}{l}N \\
\text { nे }\end{array}$ & $\begin{array}{l}m \\
\hat{n}\end{array}$ & $\begin{array}{l}\text { जे } \\
\text { के }\end{array}$ & in & $\begin{array}{l}0 \\
\text { के }\end{array}$ & $\hat{i}$ & $\begin{array}{l}\infty \\
\hat{n}\end{array}$ & iे & $\begin{array}{l}\frac{0}{2} \\
\text { in }\end{array}$ & $\begin{array}{l}\frac{5}{5} \\
\text { nे }\end{array}$ & $\begin{array}{l}\frac{1}{2} \\
\text { ì }\end{array}$ & $\begin{array}{l}m \\
\text { ì }\end{array}$ & $\begin{array}{l}j \\
\text { iे }\end{array}$ & $\begin{array}{l}\text { n? } \\
\text { ஸे }\end{array}$ & 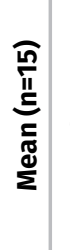 & जे & $\frac{\mathrm{c}}{\frac{\pi}{\pi}}$ & 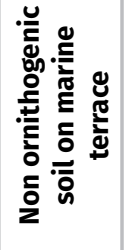 & 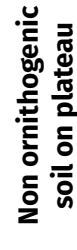 \\
\hline
\end{tabular}




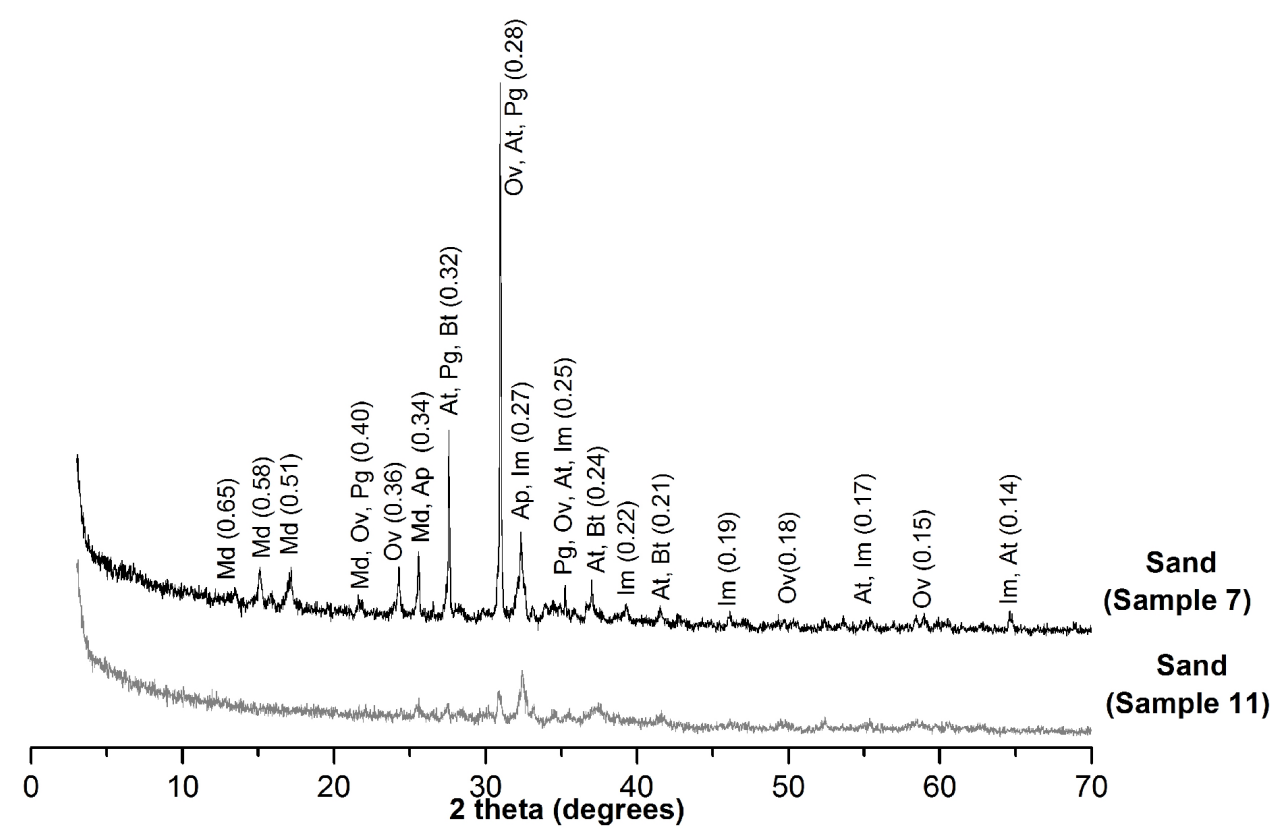

Figure 2. Representative XRD of sand samples of ornithogenic sites (samples 7 and 11). Md - mordenite; Ov - olivine; Bt - biotite; $\mathrm{Pg}$ - plagioclase; At augite; Im - ilmenite; Ap - apatite). "d" in nm.

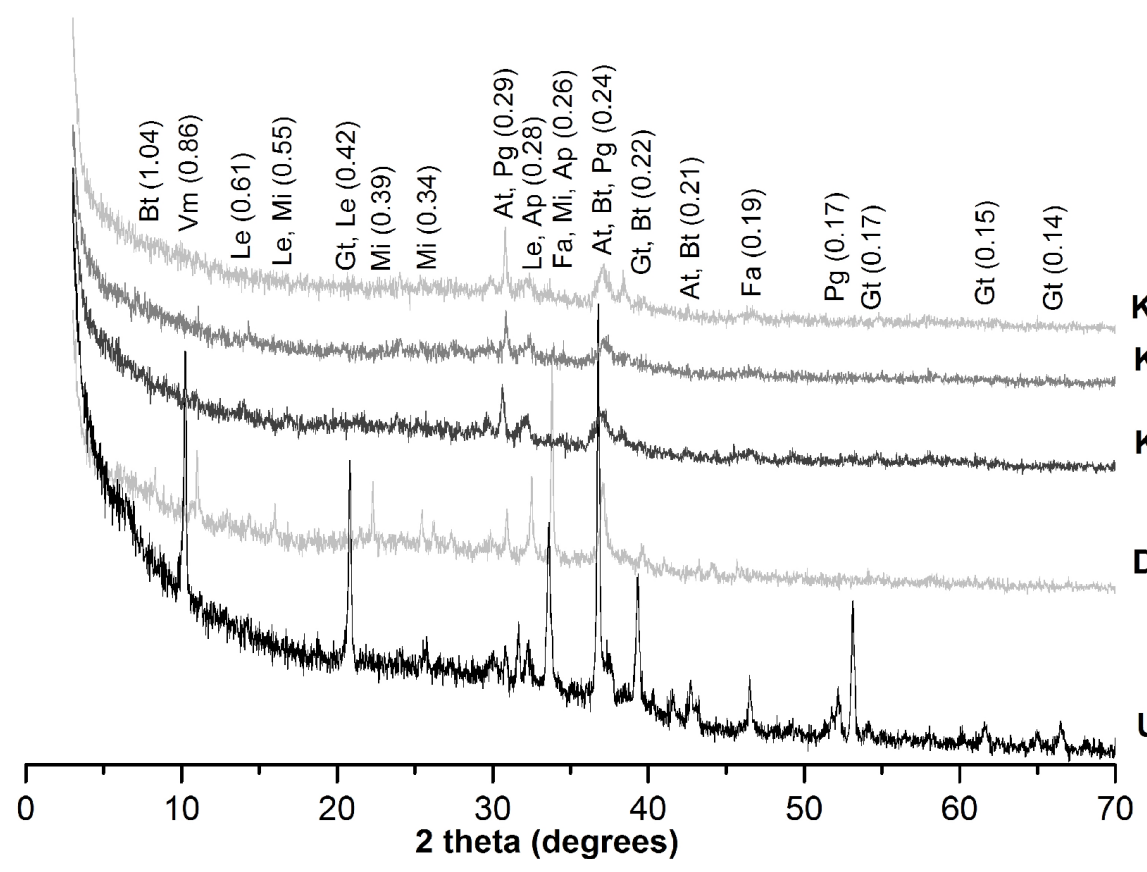

Figure 3. Representative XRD of clay sample of ornithogenic soil (sample 7). Unt. Clay - Untreated clay; DCB - removal of iron oxides; $\mathrm{K}-\mathrm{K}^{+}$saturation (heat treatment of K-saturated mounts to $25^{\circ}, 350^{\circ}$ and $550^{\circ} \mathrm{C}$. Vm - vermiculite; $\mathrm{Bt}$ biotite; Pg - plagioclase; Le - leucophosphite; Mi - minyulite; Gt goethite; At - augite; $\mathrm{Fa}$ - fluorapatite; $\mathrm{Ap}$ apatite). "d" in $\mathrm{nm}$.

Unt. clay

Micromorphologically (Figure 5), the phosphatized soil pockets have well separated blocks and/or moderately separated planar microstructures. The groundmass consists of fragments of igneous rocks (andesitic basalts), feathers and bone apatite fragments as coarse material, immersed in a yellowish-brown P-rich micromass, with a porphyric relative distribution. Coatings and infillings features are common within pores and around rock fragments. These features have a phosphatic composition, yellowish-red colors, crescent b-fabric, like those observed by Simas et al. (2007) on King George Island. 
Table III. Descriptive statistics considering phosphatizated sites affected by flying birds comparison with penguins.

\begin{tabular}{|c|c|c|c|c|c|c|c|c|c|c|c|}
\hline \multirow{2}{*}{ Variables } & \multirow{2}{*}{ Units } & \multirow{2}{*}{$\mathbf{n}$} & \multirow{2}{*}{ Mean } & \multirow{2}{*}{ Median } & \multirow{2}{*}{ Minimum } & \multirow{2}{*}{ Maximum } & \multirow{2}{*}{$\begin{array}{c}\text { Percentile } \\
10.00000\end{array}$} & \multirow{2}{*}{$\begin{array}{l}\text { Percentile } \\
90.00000\end{array}$} & \multirow{2}{*}{$\begin{array}{l}\text { Coef. } \\
\text { Var. }\end{array}$} & \multirow{2}{*}{ Skewness } & \multirow{2}{*}{ Kurtosis } \\
\hline & & & & & & & & & & & \\
\hline \multicolumn{12}{|c|}{ Snow Island (flying birds) } \\
\hline Silt & kg.kg & 15 & 16.85 & 15.60 & 3.80 & 39.10 & 6.10 & 26.90 & 55.29 & 0.78 & 0.83 \\
\hline Clay & kg.kg & 15 & 20.03 & 19.30 & 9.10 & 29.60 & 11.10 & 27.10 & 28.51 & -0.27 & -0.28 \\
\hline Sand & kg.kg & 15 & 63.13 & 63.10 & 33.80 & 85.10 & 48.40 & 83.20 & 21.33 & -0.30 & 0.35 \\
\hline pH water & - & 15 & 5.00 & 4.83 & 4.16 & 5.90 & 4.27 & 5.83 & 11.78 & 0.18 & -1.35 \\
\hline $\mathbf{P}$ & mg.dm³ & 15 & 4503.67 & 5159.80 & 474.90 & 7092.10 & 530.30 & 7072.00 & 51.52 & -0.81 & -0.64 \\
\hline $\mathbf{K}$ & mg.dm³ & 15 & 278.87 & 269.00 & 151.00 & 468.00 & 178.00 & 388.00 & 30.17 & 0.68 & 0.43 \\
\hline $\mathrm{Na}$ & mg.dm ${ }^{3}$ & 15 & 739.23 & 759.60 & 373.50 & 1226.90 & 393.80 & 1084.70 & 39.68 & 0.17 & -1.46 \\
\hline $\mathrm{Ca}$ & cmolc.dm ${ }^{3}$ & 15 & 7.46 & 7.65 & 1.59 & 13.80 & 3.88 & 11.54 & 43.87 & 0.21 & -0.27 \\
\hline Mg & cmolc.dm ${ }^{3}$ & 15 & 3.11 & 3.06 & 0.82 & 6.65 & 1.28 & 5.42 & 53.44 & 0.65 & -0.13 \\
\hline Al & cmolc.dm $^{3}$ & 15 & 0.32 & 0.20 & 0.00 & 1.09 & 0.00 & 1.09 & 110.97 & 1.36 & 1.11 \\
\hline $\mathrm{H}+\mathrm{Al}$ & cmolc.dm $^{3}$ & 15 & 11.78 & 11.10 & 5.20 & 20.90 & 6.20 & 19.20 & 38.65 & 0.76 & -0.02 \\
\hline SB & cmolc.dm ${ }^{3}$ & 15 & 14.49 & 13.99 & 4.49 & 23.69 & 7.66 & 21.52 & 37.22 & -0.07 & -0.46 \\
\hline CECpot & cmolc.dm $^{3}$ & 15 & 26.27 & 26.67 & 13.79 & 44.59 & 18.77 & 32.62 & 26.24 & 0.94 & 3.22 \\
\hline PSB & $\%$ & 15 & 54.59 & 57.40 & 31.00 & 77.30 & 32.60 & 72.30 & 26.72 & -0.33 & -0.98 \\
\hline Al-sat & $\%$ & 15 & 3.40 & 1.30 & 0.00 & 19.50 & 0.00 & 11.20 & 156.27 & 2.44 & 6.10 \\
\hline OC & dag.Kg & 15 & 14.92 & 9.64 & 2.93 & 40.57 & 3.86 & 32.59 & 79.51 & 1.20 & 0.02 \\
\hline \multicolumn{12}{|c|}{ Penguim nesting areas in Antarctica* } \\
\hline Silt & kg.kg & 17 & 20.59 & 21.00 & 2.00 & 38.00 & 6.00 & 38.00 & 54.17 & 0.19 & -0.91 \\
\hline Clay & kg.kg & 17 & 15.76 & 15.00 & 4.00 & 39.00 & 5.00 & 32.00 & 57.33 & 1.25 & 1.82 \\
\hline Sand & kg.kg & 17 & 63.65 & 62.00 & 24.00 & 93.00 & 42.00 & 85.00 & 28.49 & -0.39 & -0.15 \\
\hline pH water & - & 17 & 5.41 & 5.31 & 4.20 & 6.72 & 4.61 & 6.59 & 13.36 & 0.30 & -0.70 \\
\hline $\mathbf{P}$ & mg.dm ${ }^{3}$ & 17 & 2636.14 & 819.90 & 174.70 & 8675.80 & 185.30 & 8326.50 & 125.83 & 1.09 & -0.69 \\
\hline $\mathbf{K}$ & mg.dm ${ }^{3}$ & 17 & 208.28 & 148.20 & 10.00 & 1134.90 & 74.00 & 347.10 & 120.97 & 3.45 & 13.08 \\
\hline $\mathrm{Na}$ & mg.dm ${ }^{3}$ & 17 & 431.70 & 275.88 & 52.80 & 1269.00 & 117.10 & 1220.70 & 85.81 & 1.45 & 1.13 \\
\hline $\mathrm{Ca}$ & cmolc.dm $^{3}$ & 17 & 4.79 & 3.32 & 0.60 & 15.42 & 0.64 & 11.40 & 93.44 & 1.19 & 0.35 \\
\hline Mg & cmolc.dm ${ }^{3}$ & 17 & 2.59 & 1.70 & 0.27 & 7.30 & 0.41 & 6.60 & 85.45 & 0.99 & -0.17 \\
\hline Al & cmolc.dm ${ }^{3}$ & 17 & 1.62 & 0.67 & 0.00 & 6.80 & 0.00 & 5.60 & 126.42 & 1.55 & 1.71 \\
\hline $\mathbf{H}+\mathbf{A l}$ & cmolc.dm ${ }^{3}$ & 17 & 14.37 & 13.70 & 4.60 & 23.80 & 6.30 & 23.30 & 49.03 & 0.04 & -1.78 \\
\hline SB & cmolc.dm ${ }^{3}$ & 17 & 9.85 & 7.40 & 1.13 & 22.72 & 1.83 & 21.80 & 70.79 & 0.63 & -0.89 \\
\hline CECpot & cmolc.dm $^{3}$ & 17 & 21.65 & 19.53 & 7.68 & 37.42 & 9.56 & 36.14 & 43.53 & 0.33 & -1.06 \\
\hline PSB & $\%$ & 17 & 40.15 & 39.20 & 4.80 & 83.20 & 9.40 & 72.20 & 58.12 & 0.23 & -0.78 \\
\hline Alsat & $\%$ & 17 & 20.95 & 6.00 & 0.00 & 72.80 & 0.00 & 71.10 & 123.88 & 1.07 & -0.29 \\
\hline OC & dag.Kg & 17 & 11.97 & 8.53 & 0.90 & 55.75 & 1.75 & 26.20 & 110.00 & 2.57 & 7.78 \\
\hline
\end{tabular}

*Simas et al. (2007), Pereira et al. (2013), Simas et al. (2015) and Daher et al. (2019). 


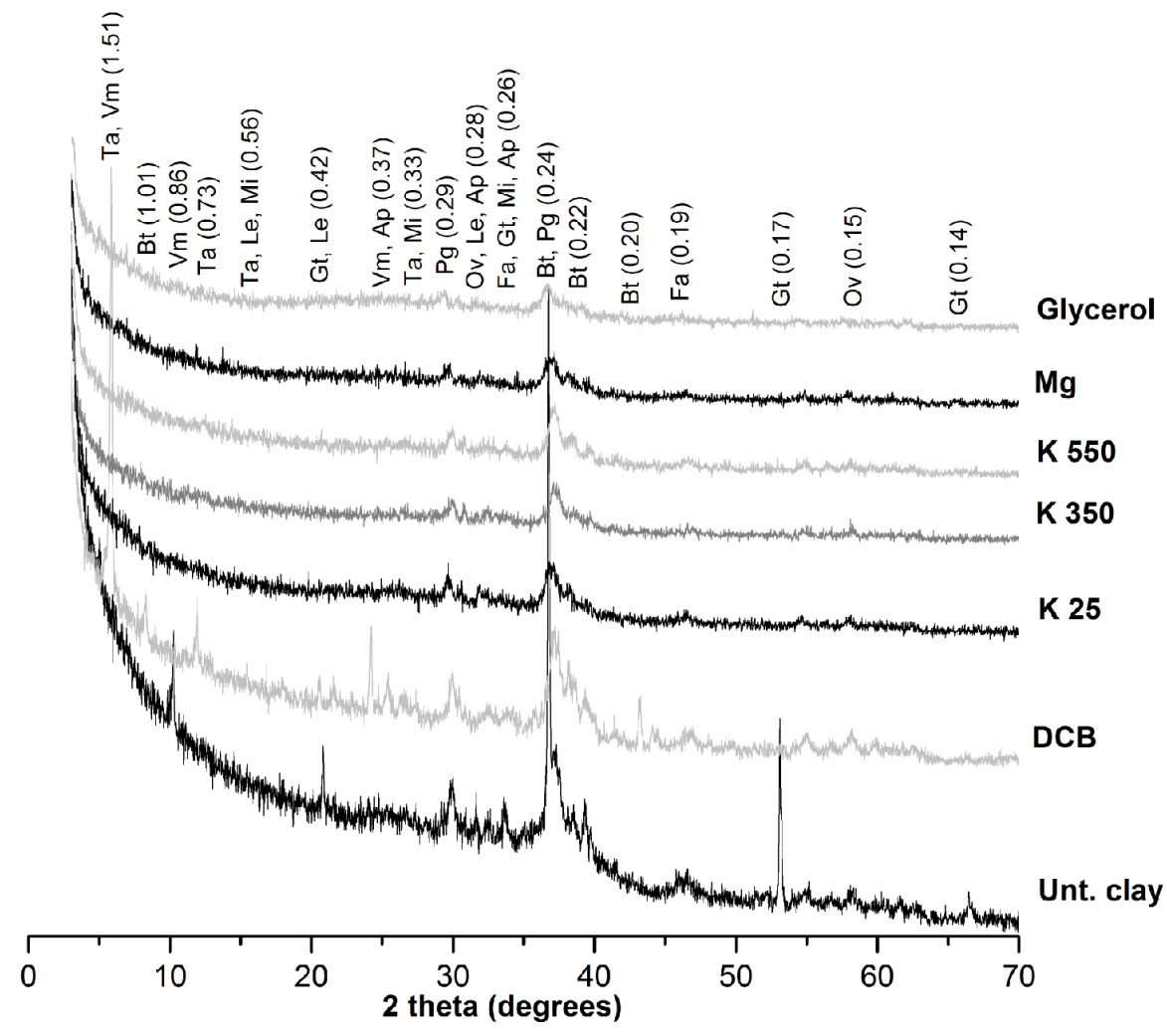

Figure 4. Representative XRD of clay sample of ornithogenic soil (sample 11). Unt. Clay - Untreated clay; DCB - removal of iron oxides; $\mathrm{K}-\mathrm{K}^{+}$saturation (heat treatment of K-saturated mounts to $25^{\circ}, 350^{\circ}$ and $550^{\circ} \mathrm{C}$; $\mathrm{Mg}-\mathrm{Mg}^{2+}$ saturation; Glycerol - saturated by $\mathrm{Mg}^{2+}$ and solvated by glycerol. Vm - vermiculite; Bt - biotite; Pg - plagioclase; Le - leucophosphite; Mi minyulite; Gt - goethite; Fa - fluorapatite; Ov - olivine; Ap - apatite; Ta - taranakite. "d" in $\mathrm{nm}$.
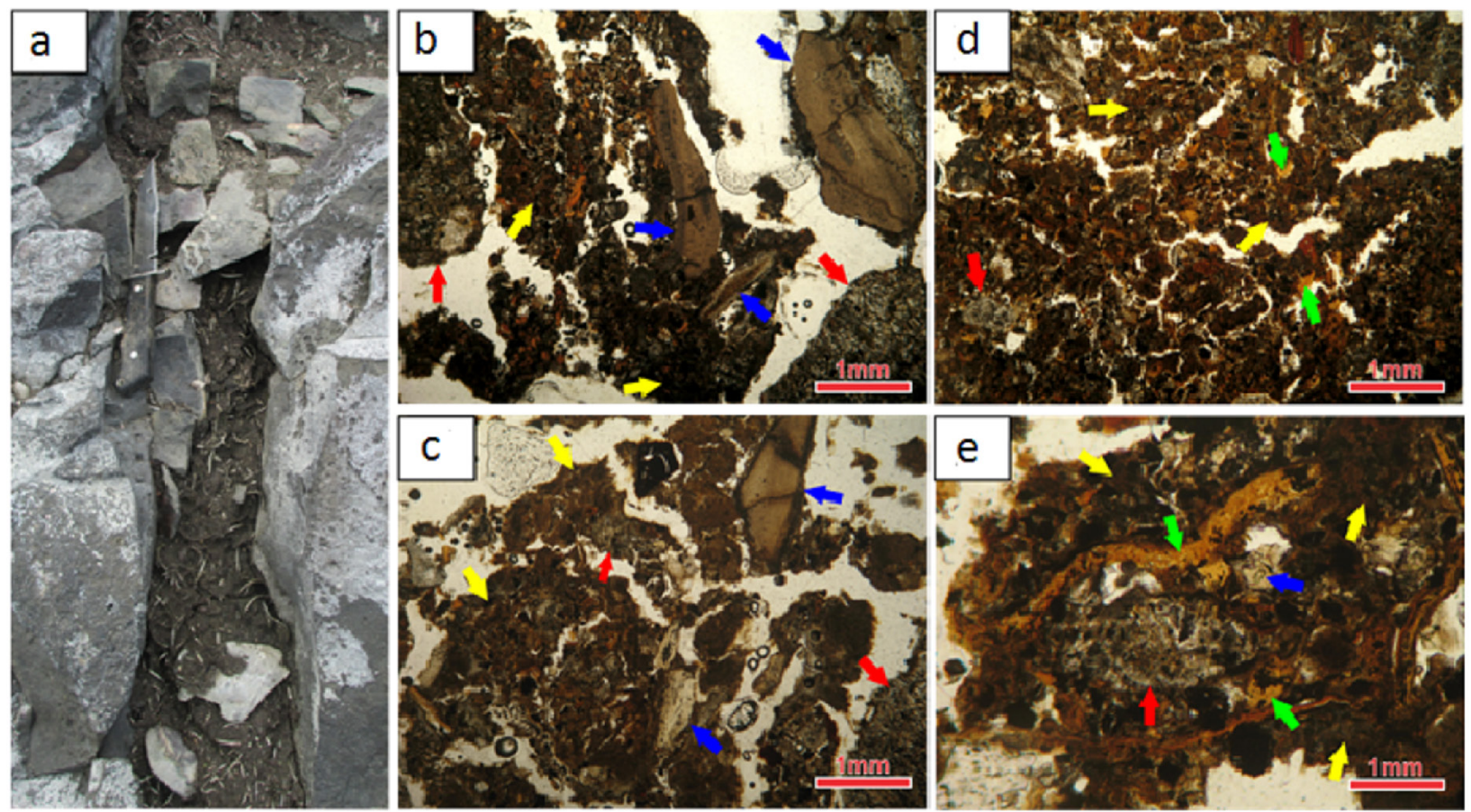

Figure 5. a) Macromorphological aspect and b, c, d, e) Micromorphological representative photomicrographs in ppl (parallel polarized light) of soil pockets affected by flying birds (phosphatizated soils). Blue arrows indicate bone fragments. Red arrows indicate rock fragments. Yellow arrows indicate phosphatizated micromass. Green arrows indicte amorphous iluvial Fe-P features. 


\section{DISCUSSION}

\section{Phosphatization flying bird's versus penguin's}

The influence of penguins in soils from Antarctica seems to be spatially much greater than the influence of flying birds, but the potential capacity of phosphatization and chemical weathering in soils affected by flying birds compared with penguins is unkonw.

The worldwide seabird population is estimated to be 804 million individuals (Otero et al. 2018), distributed mainly in the polar zones and oceanic islands, but concentrated in Antarctica and sub-Antarctic islands. The largest order is Procellariiformes, that comprises the family of petrels, with 424 million, followed by Charadriiformes, that comprises Stercorariidae/ skuas with 291 million (Otero et al. 2018). These two birds are key species on rocky terrestrial environments in Antarctica.

The phosphatization is more intense in the southern hemisphere than the northern hemisphere, because differences in body masses and length of the breeding seasons produce greater nutrient excretions (Otero et al. 2018). An essential portion of the species present in Antarctica and its sub-Antarctic islands are large-sized and heary weight (e.g. Pygoscelis antarcticus, 3-5 kg) (Otero et al. 2018). In PHP, the penguins soils showed the higher coefficient of variation (Table III) compared with soil pockets by flying birds, probably due to a greater dispersion of penguins at a single rookery, forming patches of different $\mathrm{P}$ concentration.

The maximum values of $P$ and $O C$ were recorded in penguin soils (8675.80 $\mathrm{mg} \cdot \mathrm{dm}^{3}$ and 55.75 dag.kg, respectively) (Table III). The breeding activity of penguins yields a key ecological effect at the coast zone of Maritime Antarctic (Tatur 2002) (Figures 6 and 7). The amount of daily dry excreta deposited by penguins on the land can be 46 higher than flying birds (Tatur 2002). In
Barrientos Island, Daher et al. (2019) reported a lower level of $\mathrm{P}$ in a soil pocket collected from an old giant petrel's nest, where the intensity of organic deposition was much lower compared with the active penguin soil from the vicinity.

Although the maximun values of $P$ and $O C$ were recorded in penguin soils, in our study the mean and median values of available $P$ was higher in flying birds soil pockets (median of $5159.80 \mathrm{mg} . \mathrm{dm}^{3}$ of $\mathrm{P}$ and 9.64 dag.kg of OC) (Table III). These results can be explained by the concentrated of guano deposition in flying birds nests (locally hotspots for nutrients). Penguin activity leads to the formation of greater soil development in Antarctica with deep mixing of bird detritus and granular structure (Simas et al. 2007, Daher et al. 2019, Lopes et al. 2019). These conditions promote more deeper mobilization of guano horizontally and vertically compared to soil pockets by flying birds, where guano is sheltered in closed fractures and microdepressions, where leaching is reduced (Figure 6). In nunataks ecosystems of Antarctica, Cocks et al. (1999) reported the highest levels of nutrients were associated with the Petrel's nest itself, elevated levels still occurred at $1 \mathrm{~m}$ from the nest but dropped to levels similar to those of non-ornithogenic soils at $2 \mathrm{~m}$ or $5 \mathrm{~m}$ from the nest. Hence, our results have broad implications for understanding the importance of chemical weathering on rocky outcrops subjected to ornithogenis in periglacial areas affected by flying birds.

Descriptive statistics demonstrate that soil pockets under flying birds activity show higher mean and median values of clay, P, K, Ca, Mg, BS, CEC, and OC compared to soils affected by penguin activity (Table III). The highest amount of nutrients in flying birds probably due the concentrated of guano deposition in microdepression relief on coastal outcrops (Figure 6). Chemical weathering is locally 


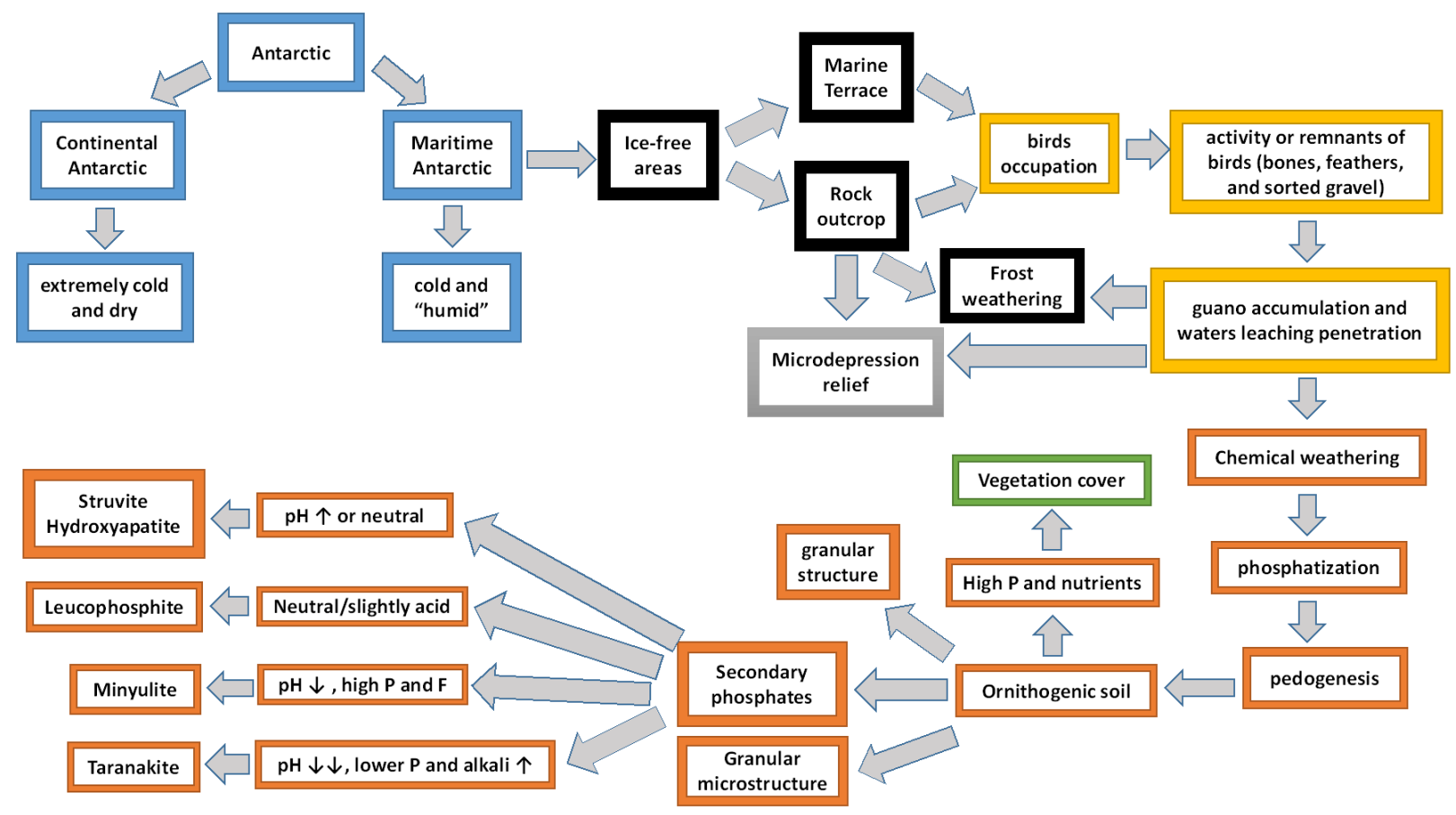

Figure 6. Integrated scheme of ornithogenic soil formation in Maritime Antarctica, the mineral assemblage representative of phosphatization and pedoenvironmental features. Blue color indicates association with climate conditions, black association with lithology, gray association with relief, yellow association with faunal activities, orange association with soil and green association with plants. Based on Tatur \& Myrcha (1984), Simas et al. (2007), Schaefer et al. (2008) and Pereira et al. (2013).

impacted by concentrated guano addition (Lopes et al. 2021a), as can be confirmed by high clay content (median of $19.3 \mathrm{~kg} . \mathrm{kg}$ ) (Table III). In this regard, the clay content was the highest in more phosphatized and acid sites with the highest amounts of $P$ in Barrientos Island, Antarctica (Daher et al. 2019).

The $\mathrm{pH}$ values on ornithogenic soils affected by penguins are similar to others reported from elsewhere in Maritime Antarctica (Michel et al. 2006, Simas et al. 2007, Pereira et al. 2013), with a mean of 5.41 (Table III), slightly higher than values reported in sites affected by flying birds in PHP, with mean of 5.00 (Table III). The statistical data demonstrated higher values Al, $\mathrm{H}+\mathrm{Al}$, and $\mathrm{Al}$ saturation for penguins soils. Guano is initially alkaline, but progressive acidification occurs, folloing the degradation of fresh guano (Pereira et al. 2013).

\section{Flying bird's phosphatization also contributes to soil mineralogical transformations}

The PHP coastal outcrops are characterized by igneous volcanic rocks. X-ray diffraction patterns of the sand fraction show the presence of mordenite (usually found in andesite and basalt), olivine, biotite, plagioclase, augite, ilmenite, and traces of apatite (Figure 2). Lavas and hypabyssal intrusions are petrografically similar, aphyric rocks contain plagioclase (andesite), olivine (occurs only in the basalts and basaltic andesites), and augite (pyroxene mineral) at Snow Island (Smellie et al. 1984). Grains of ilmenite and rare apatite are described in the petrographic analysis presented by Smellie et al. (1984). Almost all igneous rocks contain apatite as phosphate as an accessory minerals (Nash 1984). 


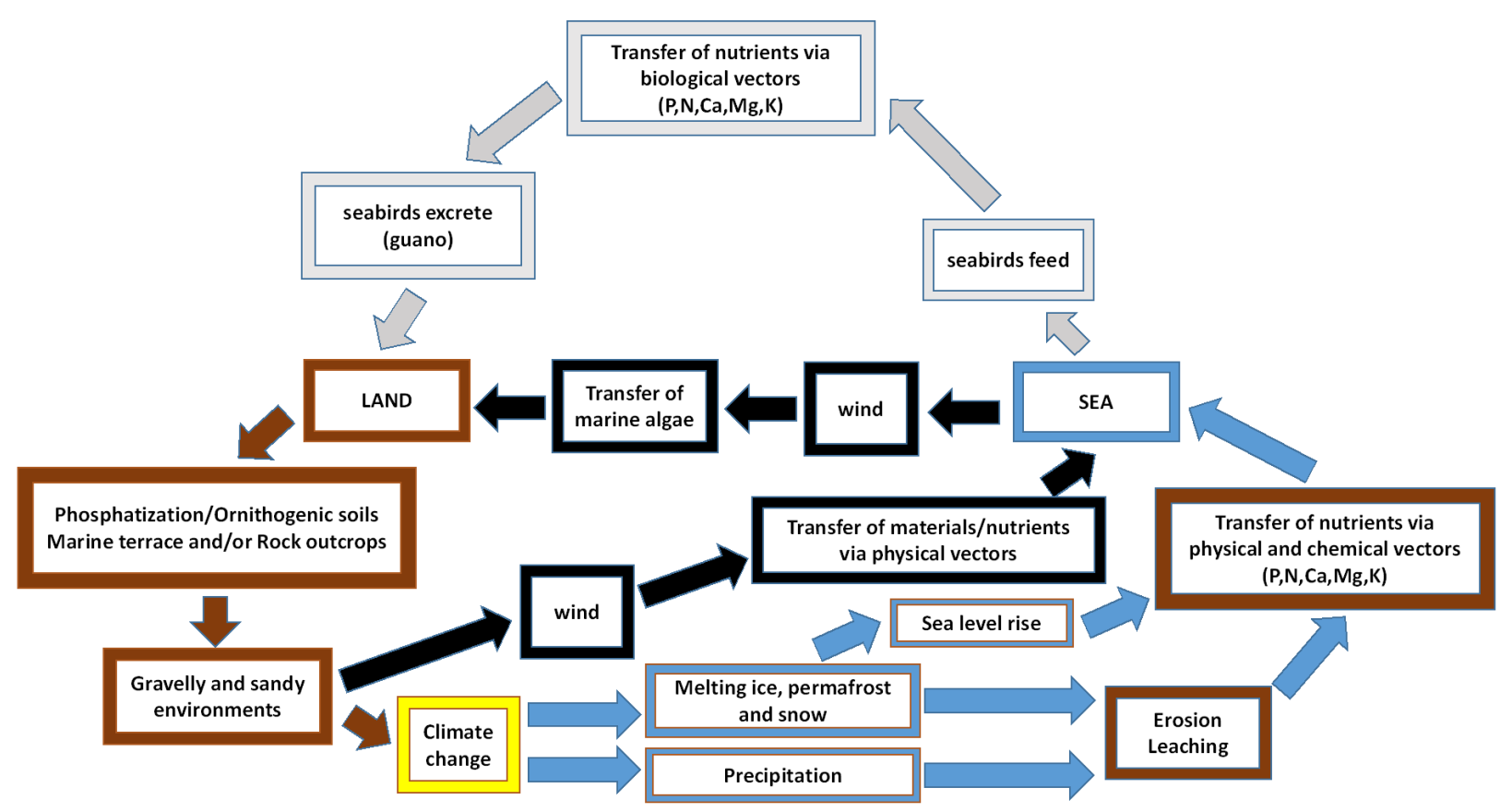

Figure 7. A proposal model of sea-land nutrient transfer in Maritime Antarctica, using climate change scenario, biological, physical and chemical vectors. Blue color indicates association with water, black association wind, gray association with seabirds, yellow association with climate and brown association with land. Informations extraction from Simas et al. (2007), Qin et al. (2014), Schaefer et al. (2017) and Otero et al. (2018).

In soil-rock contact zone, phosphatization leads to intense alteration of the lithomargin, resulting in the neoformation of phosphatization products in situ, as secondary minerals, although fragments of unaltered minerals are preserved (Tatur \& Myrcha 1984) (Figure 5). Preserved minerals in rock fragments are also observed in Snow Island by minerals like olivine, plagioclase, and biotite. In the studie soil pockets from Snow Island, we found a mixture of three phosphates with silicates in every sample. The presence of different phosphates proves varying physical and chemical conditions during phosphatization (Tatur \& Myrcha, 1984).

The phosphate assemblage on Snow Island includes leucophosphite, minyulite, fluorapatite, and apatite, confirming a highly active chemical weathering environment, by input by flying birds (Figure 6). A mineral assemblage representative of phosphatization was reported in several recent studies in coastal areas of Antarctica, but always associated with penguin activity (Tatur \& Myrcha 1984, Simas et al. 2007, Schaefer et al. 2008, Pereira et al. 2013). Slight differences may occur regarding the formation and degradation of particular phosphate minerals like the geochemical nature of the parent rock, parent rock porosity, and local landforms (Flicoteaux \& Lucas 1984) (Figure 6).

The most important phosphate in the guano layer is hydroxyapatite, besides struvite (magnesium ammonium phosphate), the latter commonly precipitated from concentrated solutions, both are stable minerals under neutral or alkaline reaction of soils (Tatur \& Myrcha 1984) (Figure 6). Struvite was not detected in Snow Island, where ornithogenic soil pockets have an acidic reaction with $\mathrm{pH}$ (water) ranging from 4.2 to 5.9 (Table II). Confirming this pattern, struvite was detected in soils from Hope Bay, 
Antarctic Peninsula, semiarid soils, with slow degradation of organic compounds and higher mean pH value of 6.1 (Pereira et al. 2013).

Once guano deposition occurs, the ornithogenic $P$ is expected to react first with amorphous aluminosilicates due to their large surface area and high P affinity (Simas et al. 2007). Soils of the South Shetland Islands are considered weakly developed, and chemical weathering processes are considered to be reduced, except in ornithogenic environments (Haus et al. 2016). The presence of weatherable primary minerals (such as plagioclases detected in the clay fraction) and secondary minerals (such as phosphates and iron hydroxides) (Figures 3 and 4) indicate that chemical weathering is not advanced in these soils at the coastal zones of Antarctica. Soil acidification favors the transformation of weatherable mafic minerals and releases highly reactive, amorphous $\mathrm{Al} / \mathrm{Fe}$ minerals, which further react with ornithogenic $\mathrm{P}$ to form amorphous and crystalline $\mathrm{Al} / \mathrm{Fe}$ phosphate minerals (Simas et al. 2007) (Figure 5).

\section{Ornithogenic environment and impacts of the phosphatization processes}

The ornithogenic environment can be considered a natural landscape unit and exhibits well-defined attributes (Figure 7), significantly distinct from the surrounding areas. The comparison of morphological, physical, chemical, and mineralogical properties between non-ornithogenic and ornithogenic soils allows a better understanding of the impacts of the faunal activity on the Antarctic terrestrial ecosystems (Simas et al. 2007).

Phosphorus from seabirds guano influences the terrestrial and freshwater ecosystems of Antarctica (Qin et al. 2014). Fresh feces, under the influence of melting and rains, are partly washed back to sea, but leachates of guano interact with the coarse soil matrix, forming a broad zone of phosphatization (Tatur \& Myrcha 1984, Lopes et al. 2021a) (Figure 7). In Snow Island, the zone of phosphatization has higher concentration of $P$ (37.9 times higher than non-ornithogenic soils on the marine terrace), $\mathrm{K}$ (2.2 times higher), and Ca (2.0 times higher) (Table II). Clay values are 1.65 higher than non-ornithogenic soils (Table I), suggesting an association between clay formation and chemical weathering promoted by the phosphatization process.

Guano deposits contain phosphates and oxalic acid, causing phosphatization and/or dissolution of silicates (Haus et al. 2016) (Figure 6). A layer of mineralized guano, rich in calcium phosphates, occurs in these areas (Tatur \& Myrcha 1989, Lopes et al. 2021a). The mean pH for the ornithogenic soils studied in the present work is 5.0, which is lower than the mean $\mathrm{pH}$ of the non-ornithogenic soils on the plateau (6.0) (Table II).

In Antarctica, high amounts of organic matter is only accumulated in coastal ornithogenic soils (Tatur \& Myrcha 1989). In the present study, organic carbon is 5.7 times higher than non-ornithogenic soils on the plateau. The high concentration of $\mathrm{P}, \mathrm{K}, \mathrm{Ca}$, and organic matter favors vegetation development in ornithogenic soils at Maritime Antarctica (Simas et al. 2007, Daher et al. 2019, Lopes et al. 2019) (Figure 6). Otero et al. (2018) estimated worldwide fluxes of total nitrogen ( $\mathrm{N}$ ) and total phosphorus (P) excreted by seabirds to be $591 \mathrm{Gg} \mathrm{N} \mathrm{y}{ }^{-1}$ and 99 Gg P $\mathrm{y}^{-1}$, with the Antarctic and Southern coasts receiving the highest $\mathrm{N}$ and $\mathrm{P}$ inputs.

High levels of $\mathrm{Na}^{+}$in ornithogenic soils (mean $739.2 \mathrm{mg} \mathrm{dm}^{-3}$ ) are attributed to saltsprays brought by winds (Daher et al. 2019). $\mathrm{Na}^{+}$levels in non-ornithogenic soils increased downslope from the plateau (380.0 $\mathrm{mg} \mathrm{dm}^{-3}$ ) to the coastal non-ornithogenic soil on the marine terrace (820.0 $\mathrm{mg} \mathrm{dm}^{-3}$ ) (Table II). 
Landforms regulate drainage and surface slope stability and change the nature or concentration of percolating solutions (Flicoteaux \& Lucas 1984). The rocky outcrops landforms at Antarctic coastal areas are very relevant for understanding the microscale of phosphatization processes. Chemical alteration is especially intense when guano is sheltered in closed fractures and depressions, where leaching is reduced (Figure 6). Thereby, a permanent and intense reation phosphatization occur. The phosphatization occurs most efficiently in deeper layers, notably in stony and permeable material (Tatur \& Myrcha 1984), subjected to physical breaking by freezing-thawing.

Faunal activity in coastal areas in Antarctica can induce permafrost degradation and enhanced periglacial erosion, as reported by Schaefer et al. (2017). Areas that receive large additions of seabird guano in Snow Island, in soil with high contents of gravel and sand (Table I) are prone to high erosion rates (Figure 7). Taking the above into account, global warming can remobilize nutrients stocked in ornithogenic environments returning them to the sea, by increasing erosion due to permafrost thawing and sea-level rise (Figure 7), as well as to a presumable increase in pluvial precipitation in Antarctic or sub-Antarctic ecosystems (Otero et al. 2018).

\section{CONCLUSIONS}

1. This study demonstrates that even under typical periglacial conditions in Antarctica, local, small soil pockets influenced by flying birds present active chemical weathering processes, such as phosphatization, and high chemical fertility.

2. The phosphatization is a chemical processes that release exchangeable bases and accelerate mineralogical and micromorphological transformations in soils, even under periglacial conditions. Despite their reduced geographic extension, these sites are important hotspots of nutrients and vegetation micro-oasis in Antarctic terrestrial ecosystems.

3. Phosphatization is a key soil-forming process in Antarctic terrestrial ecosystem resulting in changing chemical (nutritional). Formation of secondary minerals in soil pockets at Snow Island result from unusual chemical weathering processes.

4. Flying seabirds are one of the main factors influencing the vegetation establishment in rocky outcrops in Maritime Antarctica.

5. Under the current global warming trend and expected sea-level rise, the ornithogenic environments of Snow Island are susceptible to accelerated erosion rates and a great part of these hotspots may be lost for the open sea.

\section{Acknowledgments}

This work is a contribution of PPG in Geography (IGC/ UFMG) and Terrantar (DPS/UFV). We are grateful to INCT da Criosfera and PROANTAR Program for financial support and field assistance. The authors would like to thank FAPEMIG (Fundação de Amparo à Pesquisa do Estado de Minas Gerais), CAPES (Coordenação de Aperfeiçoamento de Pessoal de Nivel Superior) and CNPq (Conselho Nacional de Desenvolvimento Científico e Tecnológico) for financial support.

\section{REFERENCES}

COCKS MP, HARRIS JM, STEELE WK \& BALFOUR DA. 1999. The influence of ornithogenic products on the nutrient status of soils surrounding nests on nunataks in Dronning Maud Land, Antarctica. Polar Res 18(1): 19-26.

DAHER M, SCHAEFER CEGR, THOMAZINI A, LIMA NETO E, SOUZA CD \& LOPES DV. 2019. Ornithogenic soils on basalts from maritime Antarctica. Catena 173: 367-374. https://doi. org/10.1016/j.catena.2018.10.028.

EMBRAPA. 1997. Manual de Métodos de Análise de Solo, 2a Edição. Rio de Janeiro: Embrapa, 230 p. 
FLICOTEAUX R \& LUCAS J. 1984. Weathering of phosphate minerals. In: NRIAGU JO \& MOORE PB (Eds). Phosphate Minerals. Springer, Berlin, p. 292-317.

HAUS NW, WILHELMA KR, BOCKHEIM JG, FOURNELLE J \& MILLER M. 2016. A case for chemical weathering in soils of Hurd Peninsula, Livingston Island, South Shetland Islands, Antarctica. Geoderma 263: 185194.

IUSS WORKING GROUP WRB. 2014. World Reference Base for Soil Resources. International soil classification system for naming soils and creating legends for soil maps. Rome: World Soil Resources Reports No. 106. FAO 43: 109 p. http://scholar.google.com/scholar?hl=en\&btnG= Search \&q=intitle:World+reference+base+for+soil+resour ces+2006\#0.

LOPES DV, SCHAEFER CEGR, SOUZA JJLL, OLIVEIRA FS, SIMAS FNB, DAHER M \& GJORUP DF. 2019. Concretionary horizons, unusual pedogenetic processes and features of sulfate affected soils from Antarctica. Geoderma 347: 13-24. https: // linkinghub.elsevier.com/retrieve/pii/ S0016706117314337.

LOPES DV, SOUZA JJLL, SIMAS FNB, OLIVEIRA FS \& SCHAEFER CEGR. 2021a. Hydrogeochemistry and chemical weathering in a periglacial environment of Maritime Antarctica. Catena 197 (2021) 104959: 1-9. https://doi.org/10.1016/j. catena.2020.104959.

MICHEL RFM, SCHAEFER CEGR \& DIAS L. 2006. Ornithogenic Gelisols (Cryosols) from maritime Antarctica; pedogenesis, vegetation, and carbon studies. Soil Sci Soc Am J. 70(4): 1370-1376.

NASH WP. 1984. Phosphate minerals in terrestrial igneous and metamorphic rocks. In: NRIAGU J \& MOORE P (Eds). Phosphate Minerals. Berlin, Germany: Springer, p. 215-241.

OTERO XL, PEÑA-LASTRA S, PÉREZ-ALBERTI A, FERREIRA TO \& HUERTA-DIAZ MA. 2018. Seabird colonies as important global drivers in the nitrogen and phosphorus cycles. Nat Commun 9(1): 1-8. http://dx.doi.org/10.1038/ s41467-017-02446-8.

PEREIRA TTC, SCHAEFER CEGR, KER JC, ALMEIDA CC, ALMEIDA ICC \& PEREIRA AB. 2013. Genesis, mineralogy and ecological significance of ornithogenic soils from a semi-desert polar landscape at Hope Bay, Antarctic Peninsula. Geoderma 209-210: 98-109. http://dx.doi.org/10.1016/j. geoderma.2013.06.012.

QIN XY, SUN LG, BLAIS J, WANG YH, HUANG T, HUANG W \& XIE Z. 2014. From sea to land: assessment of the bio-transport of phosphorus by penguins in Antarctica. Chinese J. Oceanol. Limnol 32(1): 148-154.
RODRIGUES WF, OLIVEIRA FS, SCHAEFER CEGR, LEITE MGP, GAUZZI T, BOCKHEIM JG \& PUTZKE J. 2019. Soil-landscape interplays at Harmony Point, Nelson Island, Maritime Antarctica: Chemistry, mineralogy and classification. Geomorphology 336: 77-94. https://doi.org/10.1016/j. geomorph.2019.03.030.

RODRIGUES WF, OLIVEIRA FS, SCHAEFER CEGR, LEITE MGP \& PAVINATO PS. 2021. Phosphatization under birds' activity: Ornithogenesis at different scales on Antarctic Soilscapes. Geoderma 391(2021): 1-18. https://doi. org/10.1016/j.geoderma.2021.114950.

SCHAEFER CEGR, PEREIRA TTC, ALMEIDA ICC, MICHEL RF, CORRÊA GR, FIGUEIREDO LPS \& KER JC. 2017. Penguin activity modify the thermal regime of active layer in Antarctica: A case study from Hope Bay. Catena 149: 582-591. http://dx.doi. org/10.1016/j.catena.2016.07.021.

SCHAEFER CEGR, SIMAS FNB, GILKES RJC, MATHISON C, COSTA LM \& ALBUQUERQUE MA. 2008. Micromorphology and microchemistry of selected cryosols from maritime Antarctica. Geoderma 144(1-2): 104-115.

SIMAS FNB, SCHAEFER CEGR, MELO VF, ALBUQUERQUE-FILHO MR, MICHEL RF \& PEREIRA VV. 2007. Ornithogenic cryosols from Maritime Antarctica: Phosphatization as a soil forming process. Geoderma 138(3-4): 191-203.

SIMAS FNB, SCHAEFER CEGR, MELO VF, GUERRA MBB, SAUNDERS $M$ \& GILKES RJ. 2006. Clay-sized Minerals in Permafrostaffected Soils (Cryosols) from King George Island, Antarctica. Clays Clay Miner 54(6): 723-738.

SIMAS FNB, SCHAEFER CEGR, MICHEL RFM \& FRANCELINO MR. 2015. In: BOCKHEIM JG (Ed). Soils of the South Orkney and South Shetland Islands, Antarctica. Springer International Publishing, Switzerland, p. 227-273.

SMELLIE JL, PANKHURST R, THOMSON MRA \& DAVIES RES. 1984. The geology of the South Shetland Islands: VI. Stratigraphy, geochemistry and evolution. Cambridge, British Antarctic Survey, 85 p. (British Antarctic Survey Scientific Reports, 87).

SOUZA CD, SCHAEFER CEGR, SIMAS FNB, SPINOLA DN \& DAHER M. 2014. Soil formation in Seymour Island, Weddell Sea, Antarctica, Geomorphology: 1-13. http://dx.doi. org/10.1016/j.geomorph.2014.03.047.

STOOPS G. 2003. Guidelines for the analysis and description of soil and regolith thin sections. SSSA, Madison, $184 \mathrm{p}$.

STOOPS G, MARCELINO V \& MEES F. 2010. Interpretation of micromorphological features of soils and regoliths. Elsevier, 720 p. 
TATUR A. 1989. Ornithogenic soils of the maritime Antarctic The maritime Antarctic forms a climatic zone, which surrounds the. Polish Polar Res 10(4): 481-532.

TATUR A. 2002. Ornithogenic Ecosystems in the Maritime Antarctic - Formation, Development and Disintegration. In: BEYER L \& BÖLTER M. Geoecology of Antarctic Ice-free Coastal Landscapes. Berlin: Springer, p. 161-184.

TATUR A \& MYRCHA A. 1984. Ornithogenic soils on King George Island, South Shetland Islands (Maritime Antarctic Zone). Polish Polar Res 5(1-2): 31-60.

TATUR A \& MYRCHA A. 1989. Soils and vegetation in abandoned penguin rookeries (maritime Antarctic). Proceedings of the NIPR Symposium on Polar Biology 2: 181-189.

TATUR A, MYRCHA A \& NIEGODZISZ J. 1997. Formation of abandoned penguin rookery ecosystems in the maritime Antarctic. Polar Biol 17(5): 405-417.

TORRES T, BARALE G, MEÓN H, PHILIPPE M \& THEVENARD F. 1997. Cretaceous Floras from Snow Island (South Shetland Islands, Antárctica) and Their Biostratigraphic Significance. The Antarctic Region: Geological Evolution and Processes: 1023-1028.

UGOLINI FC. 1972. Orthinogenic soils of Antarctica. In: LLANO GA. Antarctic Terrestrial Biology. Washington, DC: American Geophysical Union: 181-193.

YEOMANS JC \& BREMNER JM. 1988. A rapid and precise method for routine determination of organic carbon in soil. Commun Soil Sci Plant Anal 19(13): 1467-1476. http://www. tandfonline.com/doi/abs/10.1080/00103628809368027.

\section{How to cite}

LOPES DV, DE OLIVEIRA FS, DE SOUZA JJLL, MACHADO MR \& SCHAEFER CEGR. 2022. Soil pockets phosphatization and chemical weathering of sites affected by flying birds of Maritime Antarctica. An Acad Bras Cienc 94: e20210595. DOI 10.1590/0001-3765202220210595.

\section{DAVÍ DO VALE LOPES}

https://orcid.org/0000-0003-3336-7397

\section{FÁBIO S. DE OLIVEIRA ${ }^{2}$}

https://orcid.org/0000-0002-1450-7609

\section{JOSÉ JOÃO L.L. DE SOUZA}

https://orcid.org/0000-0003-4670-6626

MARIANA DE RESENDE MACHADO ${ }^{2}$

https://orcid.org/0000-0002-1180-6363

\section{CARLOS ERNESTO G.R. SCHAEFER ${ }^{3}$}

https://orcid.org/0000-0001-7060-1598

${ }^{1}$ Universidade Federal do Rio Grande do Norte, Centro de Ensino Superior do Seridó (CERES), Departamento de Geografia, Rua Joaquim Gregório, s/n, Penedo, 59300-000 Caicó, RN, Brazil

${ }^{2}$ Universidade Federal de Minas Gerais, Departamento de Geografia, Av. Antônio Carlos, 6627, Pampulha, 31270-901 Belo Horizonte, MG, Brazil

${ }^{3}$ Universidade Federal de Viçosa, Departamento de Solos, Av. PH Rolfs, s/n, 36570-000 Viçosa, MG, Brazil

Correspondence to: Daví do Vale Lopes

E-mail:davivlopes@hotmail.com

\section{Author contributions}

Designed and study conception: DVL, FSO and CEGRS; Data gathering: DVL and FSO; Data analyses and processed: DVL and JJLLS; Material contribution: JJLLS and MRM; Manuscript main writing: DVL; Manuscript editing and review: FSO, JJLLS, MRM and CEGRS.

\section{(cc) BY}

